\title{
Phase-field modeling of crack propagation in piezoelectric and ferroelectric materials with different electromechanical crack conditions
}

\author{
Amir Abdollahi, Irene Arias* \\ Laboratori de Càlcul Numèric (LaCàN), Departament de Matemàtica Aplicada III, Universitat Politècnica de Catalunya (UPC), Campus Nord UPC-C2, \\ E-08034 Barcelona, Spain
}

\section{A R T I C L E I N F O}

\section{Article history:}

Received 15 March 2012

Received in revised form

4 June 2012

Accepted 28 June 2012

Available online 3 August 2012

Keywords:

Piezoelectricity

Ferroelectricity

Fracture

Phase-field models

Crack-face boundary conditions

\begin{abstract}
A B S T R A C T
We present a family of phase-field models for fracture in piezoelectric and ferroelectric materials. These models couple a variational formulation of brittle fracture with respectively, (1) the linear theory of piezoelectricity, and (2) a Ginzburg-Landau model of the ferroelectric microstructure to address the full complexity of the fracture phenomenon in these materials. In these models, both the cracks and the ferroelectric domain walls are represented in a diffuse way by phase-fields. The main challenge addressed here is encoding various electromechanical crack models (introduced as crack-face boundary conditions in sharp models) into the phase-field framework. The proposed models are verified through comparisons with the corresponding sharp-crack models. We also perform two dimensional finite element simulations to demonstrate the effect of the different crack-face conditions, the electromechanical loading and the media filling the crack gap on the crack propagation and the microstructure evolution. Salient features of the results are compared with experiments.
\end{abstract}

(c) 2012 Elsevier Ltd. All rights reserved.

\section{Introduction}

The design and implementation of electromechanical systems demand high performance materials regarding their coupling behavior and reliability. Piezoelectric ceramics are very prominent in this field, exhibiting strong electromechanical coupling with short response times. However, their inherent brittleness is a serious obstacle to their reliable operation in devices, which demands a deep understanding of the fracture behavior. Towards this goal, numerous theoretical and experimental investigations have been carried out during the past decades on the fracture of piezoceramics. Comprehensive reviews of these works have been presented (Zhang and Gao, 2004; Schneider, 2007; Kuna, 2010). Most piezoelectrics also exhibit ferroelectric and ferroelastic switching behavior with macroscopic dielectric and butterfly hysteresis. For related modeling approaches, see the reviews by Kamlah (2001), Landis (2004b) and Huber (2005). Microstructural domains often nucleate and evolve under high electromechanical loadings and near load concentrations such as those produced at crack tips (Hackemann and Pfeiffer, 2003; Jones et al., 2007; Pojprapai et al., 2011). The interactions between the microstructure and the localized stress and electric fields near the crack tips are responsible for

\footnotetext{
* Corresponding author. Tel.: + 34 934054181; fax: +34 934011825 .

E-mail address: irene.arias@upc.edu (I. Arias).

URL: http://www.lacan.upc.edu/arias/ (I. Arias).
} 
the complexity of fracture in ferroelectric materials. Furthermore, it is widely accepted that the crack-face boundary conditions strongly affect the electromechanical fields and thus play an important role in these interactions.

To gain theoretical understanding of fracture in these materials, researchers have resorted to the linear theory of piezoelectricity, where microstructure effects are not taken into account. Due to their simplicity, these models are useful to study the basic concepts of the linear theory in the context of fracture mechanics and to evaluate the effects of individual and coupled electromechanical fields and different crack-face boundary conditions (McMeeking, 1999, 2004; Landis, 2004a; Li et al., 2008). Such approaches have allowed researchers to identify the effect of remanent polarization on energy release rates in poled ferroelectrics (Haug and McMeeking, 2006). However, the intrinsic nature of most piezoelectric materials demands the consideration of the nonlinear microstructure effects. These include models inspired in plasticity theory and aimed at polycrystalline ferroelectric ceramics (McMeeking and Landis, 2002). A phenomenological constitutive theory has been introduced to describe implicitly the domain formation around the cracks (Landis, 2003; Wang and Landis, 2006; Sheng and Landis, 2007). Other models rely on an energy-based switching criterion (Hwang et al., 1995), considering the local phase transformations near the crack tip under the assumption of small-scale switching (Zhu and Yang, 1999, 1997; Yang and Zhu, 1998; Beom and Atlurib, 2003). Recently, phase-field or time-dependent DevonshireGinzburg-Landau (TDGL) models are gaining a growing attention. These models explicitly describe the formation and evolution of individual ferroelectric domains in the framework of continuum mechanics (Shu and Bhattacharya, 2001; Zhang and Bhattacharya, 2005; Schrade et al., 2007; Su and Landis, 2007; Xu et al., 2009), and have been extended to accurately account for the stray fields (Yang and Dayal, 2011). Related models have also been developed in micromagnetics (DeSimone et al., 2001; DeSimone and James, 2002). The nucleation and growth of domains near crack tips have been studied using these microstructural models and the influence on the stress field (Yang and Dayal, 2012) and the mechanical and electromechanical J-integrals have been reported (Song et al., 2007; Wang and Zhang, 2007; Wang and Kamlah, 2009; Xu et al., 2010b; Li and Landis, 2011; Li and Kuna, 2012). For completeness, we mention that cohesive theories aimed at fracture in ferroelectric materials have also been proposed (Gao et al., 1997; Arias et al., 2006).

The above mentioned models of ferroelectric fracture consider stationary crack configurations. With this assumption it is not possible to capture the effects of the microstructure on the crack propagation behavior, specially when the crack tip field interacts with obstacles such as defects or grain boundaries. Recently, a phase-field model (Miehe et al., 2010; Linder
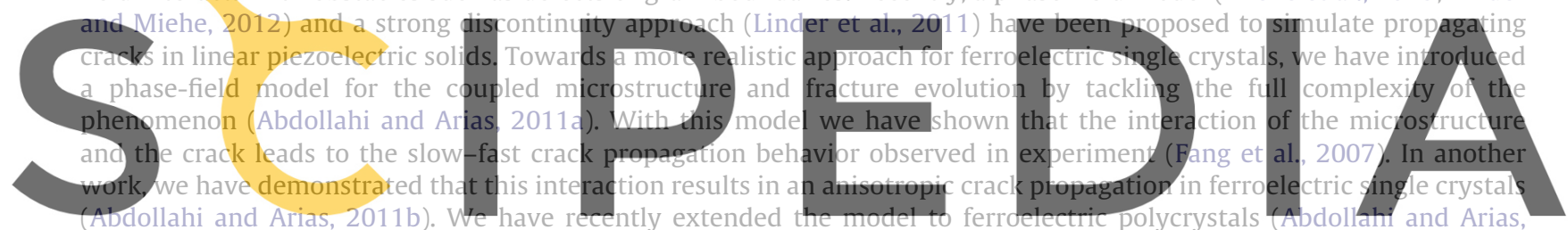

2012). We have considered so far, the simplest crack-face boundary conditions in electromechanical fracture. A similar

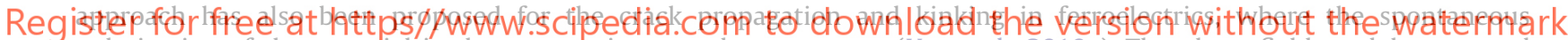
polarization of the material is chosen as primary order parameter (Xu et al., 2010a). The phase-field models proposed by Abdollahi and Arias (2011a,b, 2012) are based on variational theories of ferroelectric ceramics (Shu and Bhattacharya, 2001; Zhang and Bhattacharya, 2005; Su and Landis, 2007; Schrade et ai., 2007) and brittie fracture (Francfort and iviarigo, 1998; Bourdin et al., 2000, 2008; Bourdin, 2007; Amor et al., 2009). The variational structure of these models allows us to naturally couple multiple physics. In addition, phase-field models are particularly interesting since a single partial differential equation governing the phase-field accomplishes at once (1) the tracking of the interfaces (cracks, domain walls) in a smeared way and (2) the modeling of the interfacial phenomena such as domain-wall energies or crack-face boundary conditions. This computational approach smears both the crack and the domain wall, and treats naturally crack and domain nucleation, crack branching, crack and domain wall merging, and interactions between multiple cracks and domains. This is in contrast with the sharp interface models of fracture such as cohesive zone models (Xu and Needleman, 1994; Camacho and Ortiz, 1996), extended finite element method (XFEM) (Moes et al., 1999), or the strong discontinuity approach (Oliver et al., 2002) and ferroelectric domain evolution (Loge and Suo, 1996; Li and Liu, 2004), which require the crack surfaces and domain walls to be tracked algorithmically. On the other hand, the peridynamic approach (Silling, 2000; Dayal and Bhattacharya, 2006) can be viewed as an alternative to the phase-field approach, smearing sharp discontinuities. However, the peridynamic theory is relatively new and it needs to be developed further to address electromechanical materials.

The flexibility of phase-field models come at the expense of a high computational cost, since the width of the phasefield regularization of the domain wall and the crack must be resolved by the discretization. Another challenge, which is the main objective of this work, is to encode different sharp-crack boundary conditions into the phase-field framework. We are particularly interested in different crack-face boundary conditions because they have a strong effect on the fracture behavior of piezoelectrics and ferroelectrics, and ultimately on the reliability of the devices. For this purpose, we propose a general framework encompassing all the usual crack-face boundary conditions in the context of phase-field models. The phase-field model of brittle fracture is viewed as a regularization of Griffith's sharp-crack model. In this sense, the aim here is to converge to the corresponding sharp-crack solutions of the specific fracture problem. We consider the different crackface boundary conditions proposed in the literature for sharp-crack models in electromechanical materials and formulate them in the regularized phase-field model. Note that here the cracks are not boundaries of the computational domain but 
rather features of the solution within the domain, and hence the different sharp-crack conditions have to be modeled in the phase-field partial differential equations.

In the context of sharp-crack models in electromechanical materials, the most common crack-face boundary conditions in the literature can be classified as follows:

A. Uncoupled electrical/mechanical crack-face conditions. Mechanical boundary conditions: These are mainly: (1) tractionfree crack faces and (2) cohesive zone models (Xu and Needleman, 1994; Camacho and Ortiz, 1996) introducing a traction-separation law on the crack faces. Here, we consider only traction-free crack boundary conditions. The encoding of the cohesive crack-face conditions in the context of the phase-field models is beyond the scope of this paper.

Electrical boundary conditions: These are mainly (1) permeable, (2) impermeable and (3) semi-permeable crack models, each assuming different electrical properties of the crack gap. The permeable crack boundary conditions were the first to be considered for electromechanical materials (Parton, 1976). These conditions assume that the crack does not exist electrically, i.e. crack faces are closed and electrical fields are not perturbed by the presence of the crack. In contrast, impermeable boundary conditions were proposed to define an open and electrically defective crack by assuming zero permittivity for the crack gap (Deeg, 1980). However, both the permeable and impermeable crack boundary conditions are not physically justifiable in many cases, since the effect of the medium filling the crack gap is neglected. As an improvement, semi-permeable boundary conditions were introduced to treat the crack gap as a linear dielectric material with a finite permittivity (Hao and Shen, 1994). As a result, the electric charge can penetrate the crack gap. A physical inconsistency of the semi-permeable conditions is that the stored electric charge in the crack gap induces a closing traction on the crack faces, which is not considered in these conditions (McMeeking, 2004). This theory has been shown to be variationally inconsistent. Furthermore, the electric field inside the crack gap is limited by the dielectric strength of the crack-gap filling medium. Above this level of electric field, the medium experiences failure of its insulating properties, i.e. electrical breakdown.

B. Coupled electromechanical crack-face conditions. The Energetically Consistent (EC) crack model was proposed to overcome the inconsistency of the semi-permeable boundary conditions by considering not only the electric charge

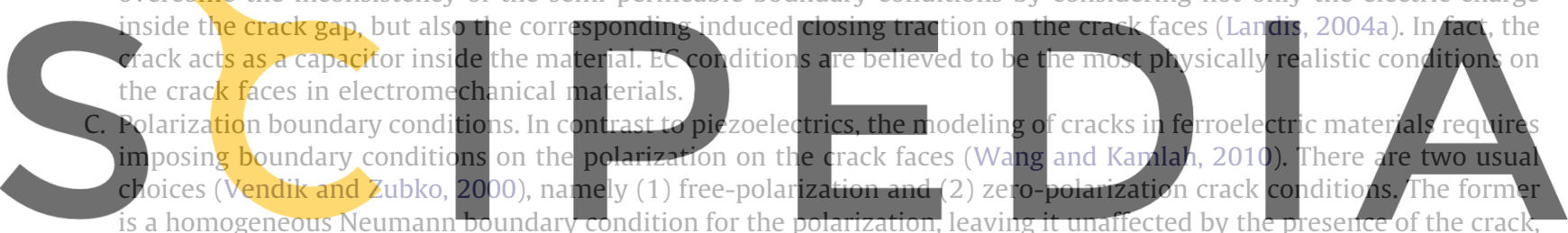

and hence dictated by the bulk material model. The latter is a homogeneous Dirichlet boundary condition for the

In the following, we first summarize in Sections 2 and 3, the phase-field model of brittle fracture and the variational formulation of ferroelectric and piezoelectric materials, respectively. Next, in Section 4 we describe the formulation of the phase-field models for fracture in these materials. We focus on the phase-field formulation of the most relevant mechanical, electrical, and electromechanical crack-face boundary conditions as described above. For each possible choice of crack-face boundary conditions, a different phase-field formulation is proposed. The corresponding governing equations are presented in Section 4.4, along with a general solution procedure. The proposed models are exercised numerically in Section 5, where the effects of the different crack-face boundary conditions, electromechanical loadings and crack-gap media on the crack propagation are evaluated. Numerical simulation results are discussed and compared with experiments. The last section is the conclusion of this paper.

\section{Phase-field modeling of fracture in brittle materials}

In the variational regularized formulation of Griffith's fracture theory first proposed by Francfort and Marigo (1998), the total energy of a body made of a brittle material and occupying a region $\Omega$ is obtained as the sum of the bulk and surface energies (Bourdin et al., 2000):

$$
E_{\kappa}[\boldsymbol{u}, v]=\int_{\Omega}\left(v^{2}+\eta_{\kappa}\right) F(\boldsymbol{\varepsilon}(\boldsymbol{u})) \mathrm{d} \Omega+G_{c} \int_{\Omega}\left[\frac{(1-v)^{2}}{4 \kappa}+\kappa|\nabla v|^{2}\right] \mathrm{d} \Omega,
$$

where $F$ is the elastic potential, $\boldsymbol{u}$ is the mechanical displacement, $\boldsymbol{\varepsilon}$ is the strain defined as $\boldsymbol{\varepsilon}(\boldsymbol{u})=1 / 2\left(\nabla \boldsymbol{u}+\nabla \boldsymbol{u}^{T}\right)$, and $v$ is a scalar phase field describing a smooth transition in space between unbroken $(v=1)$ and broken $(v=0)$ states of the material. For a linear elastic material, $F(\boldsymbol{\varepsilon})=\frac{1}{2} \boldsymbol{\varepsilon}: \mathbb{C}: \boldsymbol{\varepsilon}$, where $\mathbb{C}$ is the forth-order tensor of elastic moduli. By noting that $v^{2}$ multiplies the elastic potential $F$ in the bulk energy (first integral), it is clear that the value $v=0$ effectively reduces the stiffness of the material to zero in the fractured zone. $\eta_{\kappa}$ is a small residual stiffness to avoid the singularity of the bulk energy in this zone. In the surface energy, $G_{c}$ is the critical energy release rate or the surface energy in Griffith's theory 


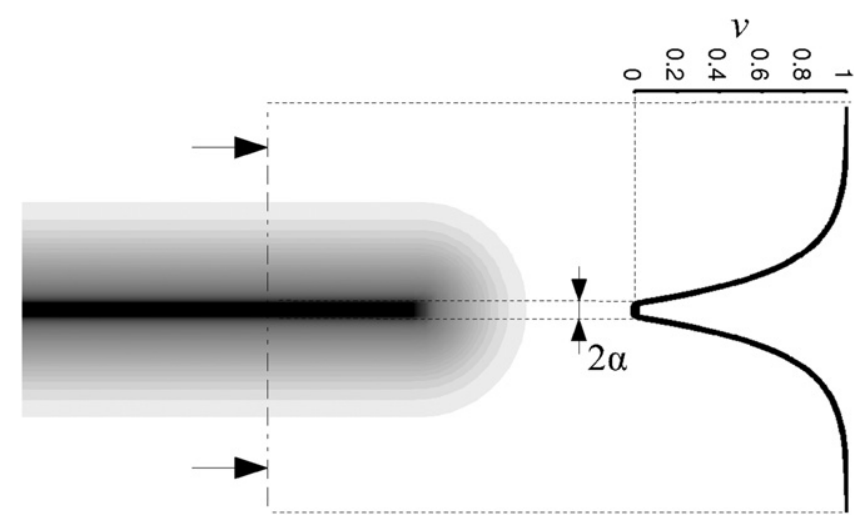

Fig. 1. Example of a smeared crack using the profile in Eq. (2).

(Griffith, 1921). $\kappa$ is a positive regularization constant, which regulates the size of the smeared fracture zone. It can be shown mathematically, by way of $\Gamma$-convergence, that when the regularization parameter $\kappa$ tends to zero, this regularized theory converges to Griffith's brittle fracture model (Bourdin et al., 2008). In particular, the traction-free conditions on the crack faces of the sharp model are recovered in the limit of a vanishingly small regularization parameter. Working by analogy, the different electrical and electromechanical conditions of the sharp-crack model in piezoelectrics and ferroelectrics are encoded into the phase-field framework in Section 4.

The crack propagation in this model results from the competition between the bulk and surface energy terms. Deformation of an elastic body under load increases the elastic energy density $F$. When this value approaches a critical value in a given region, it is energetically favorable for the system to decrease the value of $v$ towards zero in that region in order to release elastic energy. On the other hand, decreasing the value of $v$ leads to an increase in the surface energy since deviabions from 1 are penalized. formation of smeared cracks wh critical energy release rate $G_{C}$ propagate cracks. It can be show

as expected in the sharp-interface theory as outlined above may
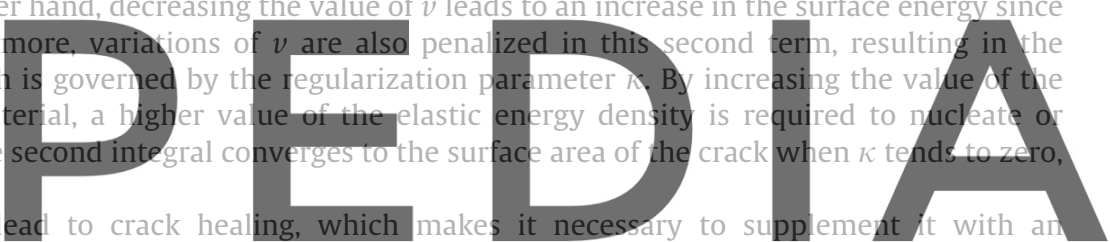

irreversibility condition. Requiring the field $v$ to be a monotonically decreasing function of time is cumbersome, and in practice, the field $v$ is frozen to 0 when and where it reaches a given small threshold $\gamma$ (Bourdin, 2007; Bourdin et al. 2008)

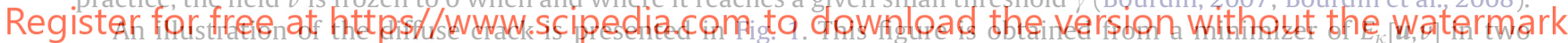

dimensions. We show the profile of $v$ perpendicular to the crack in its wake. Denoting $d(x)$ the distance to the crack of a point $x$, it can be shown that this optimal profile takes the form (Bourdin et al., 2008)

$$
v_{\kappa}(x)= \begin{cases}0 & \text { if } d(x) \leq \alpha_{\kappa} \\ 1-\exp \left(-\frac{d(x)-\alpha_{\kappa}}{2 \kappa}\right) & \text { otherwise }\end{cases}
$$

where $2 \alpha_{\kappa}$ indicates the width of the fully fractured region where $v=0$. This width is given by the threshold $\gamma$, and it is smaller than $\kappa, \alpha_{\kappa}=o(\kappa)$.

The total energy in Eq. (1) is quadratic and convex in $v$ and $\boldsymbol{u}$ separately. Therefore, for a fixed $v$ or $\boldsymbol{u}, E_{\kappa}(\boldsymbol{u}, \bullet)$ and $E_{\kappa}(\bullet, v)$ can be efficiently minimized solving a linear system of equations, with the appropriate boundary conditions. As a consequence, the numerical implementation of this theory is straightforward by means of an iterative algorithm minimizing separately each field in a staggered manner.

\section{Variational formulation of electromechanical solids}

The behavior and properties of electromechanical solids such as ferroelectric and piezoelectric materials can be defined by a thermodynamical potential containing mechanical, electrical and electromechanical coupling energy terms. The form of this potential and number of parameters depend on the complexity of the material behavior. In ferroelectric ceramics, the polarization and strain state can evolve in a nonlinear fashion due to ferroelectric/ferroelastic domain switching. However, under the assumption of a small mechanical/electrical load, domain switching cannot occur and the material behavior can be expressed by the linear theory of piezoelectricity. In fact, a piezoelectric material can be viewed as a linear approximation of the ferroelectric material behavior near the spontaneous polarization and strain state.

Ignoring body loads and volume charges for simplicity, the total electromechanical enthalpy of a ferroelectric or piezoelectric body occupying a region $\Omega$ can be written in terms of the mechanical displacement $\boldsymbol{u}$, the total polarization $\mathbf{p}$ 
and the electric potential $\phi$, as

$$
H[\boldsymbol{u}, \mathbf{p}, \phi]=\int_{\Omega} \mathcal{H}(\boldsymbol{\varepsilon}(\boldsymbol{u}), \mathbf{p}, \nabla \mathbf{p}, \boldsymbol{E}(\phi)) \mathrm{d} \Omega-\int_{\Gamma_{N, \boldsymbol{u}}} \boldsymbol{t} \cdot \boldsymbol{u} \mathrm{d} S+\int_{\Gamma_{N, \phi}} \omega \phi \mathrm{d} S,
$$

where $\boldsymbol{E}$ is the electric field defined as $\boldsymbol{E}=-\nabla \phi . \boldsymbol{t}$ and $\omega$ are the tractions and surface charge density, respectively. $\Gamma_{N, \boldsymbol{u}}$ and $\Gamma_{N, \phi}$ are the parts of the boundary of the domain $\partial \Omega$ where mechanical and electrical Neumann boundary conditions are applied.

To account for domain switching, we follow the phase-field model of ferroelectric domain evolution proposed by Zhang and Bhattacharya (2005) and Su and Landis (2007). The electromechanical enthalpy density $\mathcal{H}$ of a ferroelectric material is formulated as

$$
\mathcal{H}(\boldsymbol{\varepsilon}, \mathbf{p}, \nabla \mathbf{p}, \boldsymbol{E})=\frac{1}{2} \boldsymbol{\varepsilon}: \mathbb{C}: \boldsymbol{\varepsilon}-\boldsymbol{\varepsilon}: \mathbb{C}: \boldsymbol{\varepsilon}_{S}(\mathbf{p})+U(\nabla \mathbf{p})+\chi(\mathbf{p})-\boldsymbol{E} \cdot \mathbf{p}-\frac{\varepsilon_{0}}{2}|\boldsymbol{E}|^{2},
$$

where the eigenstrain $\varepsilon_{s}$ depends on the polarization, $U$ is the domain wall energy density penalizing sharp variations in the polarization, $\chi$ is the phase separation or Landau potential, and $\varepsilon_{0}$ is the permittivity of free space. The first term in Eq. (4) is the elastic potential and the second term is the electromechanical coupling energy density. The combination of these terms and energy function $\chi$ is the total Landau-Devonshire energy density penalizing deviations from the spontaneous polarizations and strains of the material, hence introducing the anisotropy and nonlinearity of ferroelectric materials. The detailed formulation of these energy functions and the material constants are given in Appendix A.

The electromechanical enthalpy density in Eq. (4) contains all crystallographic and domain wall information of a ferroelectric material. However, only some of this information is required to model a piezoelectric material by assuming that the nonlinear switching of the polarization between the various crystallographic orientations does not occur According to the linear theory of piezoelectricity, the electromechanical enthalpy density $\mathcal{H}$ of a piezoelectric material is written in terms of the strain and electric field alone (Tieresten, 1969)

$$
\mathcal{H}(\boldsymbol{\varepsilon}, \boldsymbol{E})=\frac{1}{2}\left(\boldsymbol{\varepsilon}-\boldsymbol{\varepsilon}_{r}\right): \mathbb{C}:\left(\boldsymbol{\varepsilon}-\boldsymbol{\varepsilon}_{r}\right)-\left(\boldsymbol{\varepsilon}-\boldsymbol{\varepsilon}_{r}\right): \mathbf{e}^{T} \cdot \boldsymbol{E}-\boldsymbol{E} \cdot \mathbf{p}_{r}-\frac{1}{2} \boldsymbol{E} \cdot \boldsymbol{K} \boldsymbol{E},
$$

where $\mathbf{e}$ is the third-order tensor of piezoelectric coupling constants, and $\boldsymbol{e}^{T}$ is such that $\left[\boldsymbol{e}^{T}\right]_{(i j) k}=[\boldsymbol{e}]_{k(i i)}$, where the brackets indigate the indices paired with s orcer dielectric tensor. The piez linearizing around the spontan $\sim_{\sigma}^{\text {The }}$ $\sigma=\frac{\partial \mathcal{H}}{\partial \varepsilon}, \quad D=-\frac{\partial \mathcal{H}}{\partial \boldsymbol{E}}$.
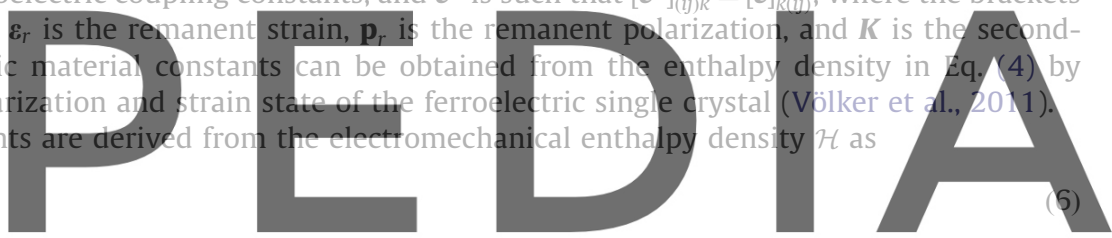

Then, given Eqs. (4) and (5), the stresses and electric displacements are obtained for the ferroelectric and piezoelectric

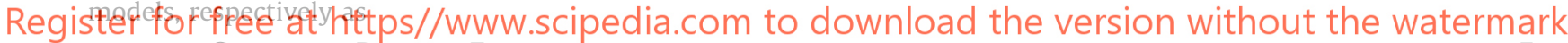
$\sigma=\mathbb{C}:\left(\varepsilon-\varepsilon_{S}\right), \quad \boldsymbol{D}=\mathbf{p}+\varepsilon_{0} \boldsymbol{E}$,

$\boldsymbol{\sigma}=\mathbb{C}:\left(\boldsymbol{\varepsilon}-\boldsymbol{\varepsilon}_{r}\right)-\mathbf{e}^{T} \cdot \mathbf{E}, \quad \boldsymbol{D}=\mathbf{p}_{r}+\boldsymbol{K E}+\mathbf{e}:\left(\boldsymbol{\varepsilon}-\varepsilon_{r}\right)$

The weak form of the mechanical and electrostatic equilibrium equations is then obtained as

$$
0=\delta H[\boldsymbol{u}, \mathbf{p}, \phi ; \delta \boldsymbol{u}, \delta \phi]=\int_{\Omega} \sigma_{j i} \delta \varepsilon_{i j} \mathrm{~d} \Omega-\int_{\Omega} D_{i} \delta E_{i} \mathrm{~d} \Omega-\int_{\Gamma_{N, u}} t_{i} \delta u_{i} \mathrm{~d} S+\int_{\Gamma_{N, \phi}} \omega \delta \phi \mathrm{d} S .
$$

The polarization evolution in ferroelectric materials is generally obtained from a gradient flow of the total electromechanical enthalpy with respect to the polarization (Zhang and Bhattacharya, 2005),

$$
\mu_{p} \int_{\Omega} \dot{p_{i}} \delta p_{i} \mathrm{~d} \Omega=-\delta H[\boldsymbol{u}, \mathbf{p}, \phi ; \delta \mathbf{p}]=-\int_{\Omega} \frac{\partial \mathcal{H}}{\partial p_{i}} \delta p_{i} \mathrm{~d} \Omega,
$$

where $1 / \mu_{p}>0$ is the mobility of the process, solved together with Eq. (9).

We take the remanent state of the piezoelectric material as the reference configuration, therefore the remanent strain $\boldsymbol{\varepsilon}_{r}$ and the remanent polarization $\mathbf{p}_{r}$ are set to zero in the following. The poling direction of the piezoelectric material is implicitly encoded in the constitutive equations. It is noteworthy that this formulation, when applied to fracture mechanics as in Section 4, introduces the implicit assumption that the remanent polarization is perfectly balanced on the crack faces. This assumption is standard in fracture mechanics studies in piezoelectric materials and, for sharp-crack models, it can be easily generalized to arbitrary levels of charge separation on the crack faces (Haug and McMeeking, 2006). Considering this assumption, the detailed formulation of Eq. (8) in Voigt form and the piezoelectric material constants are given in Appendix B.

\section{Phase-field modeling of fracture in piezoelectric and ferroelectric materials}

The specific coupling between the phase-field model of brittle fracture in Section 2 and the models of piezoelectric and ferroelectric materials in Section 3 depends on the particular choice of the crack-face conditions discussed in Section 1. 
Based on these conditions, the presence of the crack can affect different terms of the electromechanical enthalpy density $\mathcal{H}$ in Eqs. (4) and (5) through the field $v$. Therefore for each possible choice of crack-face boundary conditions a different formulation of the enthalpy density $\mathcal{H}$ is required. Sections $4.1-4.3$ deal with the uncoupled mechanical/electrical, the coupled electromechanical, and the polarization boundary conditions, respectively. Using the proposed formulations, the governing equations of the phase-field model of crack propagation in piezoelectric and ferroelectric materials are discussed in Section 4.4, along with the proposed solution algorithm.

\subsection{Uncoupled mechanical/electrical crack-face conditions}

Most electromechanical crack models assume uncoupled mechanical and electrical boundary conditions on the crack faces. Although the uncoupled conditions are not physically realistic, they are good approximations of the coupled electromechanical conditions in some situations.

\subsubsection{Traction-free conditions}

Mathematically, these conditions are stated in the context of sharp-crack models as

$$
\boldsymbol{\sigma}^{+} \cdot \boldsymbol{n}=\boldsymbol{\sigma}^{-} \cdot \boldsymbol{n}=\mathbf{0},
$$

where the superscripts + and - denote the top and bottom crack faces and $\boldsymbol{n}$ is the unit normal. In the phase-field model of brittle fracture, the traction-free conditions are encoded by multiplying the elastic energy density $F$ by the jump set function $\left(v^{2}+\eta_{k}\right)$ (see Eq. (1)). Working by analogy, in the case of the proposed electromechanical models, we multiply by the jump set function the first two terms of the enthalpy density $\mathcal{H}$ associated with the elastic strains $\boldsymbol{\varepsilon}$. Therefore, the elastic and electromechanical coupling potentials are annihilated in the fractured zone $(v=0)$.

4.1.2. Traction-free, electrically impermeable crack model

In the case of impermeable cracks, the crack faces are treated as charge-free surfaces, i.e. the normal components of the electric displacement vanish on both crack faces as

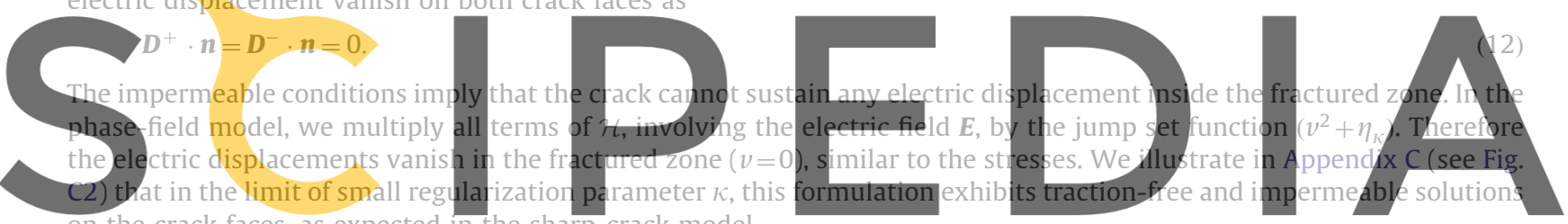

on the crack faces, as expected in the sharp-crack model.

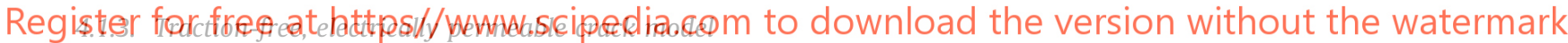 \\ The permeable crack boundary conditions imply that the electric potential and the normal component of the electric} displacement are continuous across the crack faces, i.e. in the context of sharp-crack models

$$
\phi^{+}=\phi^{-} \text {and } \boldsymbol{D}^{+} \cdot \boldsymbol{n}=\boldsymbol{D}^{-} \cdot \boldsymbol{n} .
$$

In contrast to the impermeable crack model, encoding the permeable conditions requires that all terms of the enthalpy density $\mathcal{H}$, involving the electric field $\boldsymbol{E}$, remain unmodified. As a way of example, the enthalpy density $\mathcal{H}$ of the tractionfree, electrically permeable crack model for the piezoelectric material is

$$
\mathcal{H}=\left(v^{2}+\eta_{\kappa}\right)\left(\frac{1}{2} \boldsymbol{\varepsilon}: \mathbb{C}: \boldsymbol{\varepsilon}-\boldsymbol{\varepsilon}: \mathbf{e}^{T} \cdot \boldsymbol{E}\right)-\frac{1}{2} \boldsymbol{E} \cdot \boldsymbol{K} \boldsymbol{E} .
$$

Accordingly, the electric displacements are

$$
\boldsymbol{D}=\left(v^{2}+\eta_{\kappa}\right) \mathbf{e}: \boldsymbol{\varepsilon}+\boldsymbol{K} \boldsymbol{E} .
$$

Since the traction-free conditions result in a discontinuous displacement field, Eq. (15) seems to be introducing a discontinuity in the electric displacement through the coupling term $\mathbf{e}: \boldsymbol{\varepsilon}$, in contradiction with Eq. (13). Nevertheless, we show in Appendix C (see Fig. C1) that the effect of this discontinuity in the normal direction becomes negligible when the regularization parameter $\kappa$ is small enough.

\subsection{Electromechanical crack-face conditions: energetically consistent crack model}

As described in Section 1, the semi-permeable crack conditions were introduced to model intermediate situations between the two over-simplistic extremes of a completely closed crack (permeable) and an open crack filled with a medium of negligible conductivity (impermeable). This model assumes that the crack-gap filling medium can sustain a certain degree of electric field, thereby inducing an electric displacement inside the crack. In the context of sharp-crack models, semi-permeable crack-face boundary conditions are formulated as

$$
\boldsymbol{D}^{+} \cdot \boldsymbol{n}=\boldsymbol{D}^{-} \cdot \boldsymbol{n}=D_{c}=\varepsilon_{0} E_{c},
$$


where $D_{c}$ is the induced electric displacement and $E_{c}$ is the electric field in the crack gap. It is obtained as

$$
E_{c}=-\frac{\phi^{+}-\phi^{-}}{u_{n}^{+}-u_{n}^{-}}=-\frac{\Delta \phi}{\Delta u_{n}},
$$

where the superscripts "+" and "-" denote the values on each crack face, $\Delta \phi$ is the potential drop across the crack gap and $\Delta u_{n}$ is the crack opening displacement. The main deficiency stems from the fact that the electric field inside the crack gap induces also tractions on the crack faces, which are not considered by the semi-permeable conditions. It has been demonstrated that this model is not energetically consistent (Li et al., 2008). To overcome this inconsistency, energetically consistent (EC) crack-face boundary conditions have been proposed in the context of sharp-crack models (Landis, 2004a), in which

$$
\boldsymbol{t}^{+}=\sigma_{c} \boldsymbol{n}, \boldsymbol{t}^{-}=-\sigma_{c} \boldsymbol{n},
$$

where $\boldsymbol{t}^{+}$and $\boldsymbol{t}^{-}$are the tractions acting on the crack faces and $\sigma_{c}$ is the effective stress within the crack gap. According to Landis (2004a) and assuming an infinite breakdown strength of free space, the effective stress can be expressed as

$$
\sigma_{c}=\frac{1}{2} \varepsilon_{0} E_{c}^{2} .
$$

In fact, EC conditions assume that the crack behaves electrically as a capacitor, storing electrical charge between the crack faces. The associated electrical enthalpy is given by

$$
H_{C}=\int_{S_{c}} H_{c} \Delta u_{n} \mathrm{~d} S,
$$

where $S_{c}$ denotes the surface (curve in 2D) of the sharp crack and the electrical enthalpy density $\mathcal{H}_{c}$ is

$$
\mathcal{H}_{c}=-\frac{1}{2} \varepsilon_{0} E_{c}^{2} \text {. }
$$

In proposing the electrical enthalpy in Eq. (20), it is assumed that any electric field and deformation components within the gap parallel to the crack are negligible in comparison to the normal components. In the following, we develop the general formulation of this enthalpy in the context of the phase-field models without any assumption regarding the diregtion of the electric and defor introduce geometric nonlinearity rack opening displacement. In fi

$$
\widehat{\mathcal{H}}=-\frac{\varepsilon_{0}}{2}|\widehat{E}|^{2}
$$

This enthaipy can be decomposed into the enthaipy of the intact and fracture zones using the phase-fieid $v$

$$
\widehat{\mathcal{H}}=v^{2} \widehat{\mathcal{H}}+\left(1-v^{2}\right) \widehat{\mathcal{H}}
$$

Ignoring all geometric nonlinearity in the intact zone, the total electrical enthalpy of free space becomes

$$
H_{0}=-\frac{\varepsilon_{0}}{2} \int_{\Omega} v^{2}|\nabla \phi|^{2} \mathrm{~d} \Omega-\underbrace{\frac{\varepsilon_{0}}{2} \int_{\Omega}\left(1-v^{2}\right)\left|\mathbf{F}^{-T} \nabla \phi\right|^{2} J \mathrm{~d} \Omega},
$$

$H_{c}$

where $J=\operatorname{det} \mathbf{F}$. The second term represents the phase-field counterpart of Eq. (20) and we can interpret $\widehat{\boldsymbol{E}}$ as $\boldsymbol{E}_{c}$ in the fracture zone. Fig. 2 illustrates an opening crack where the electric fields are presented both for the sharp crack across the faces and for the diffuse crack inside a partition of the fractured zone.

In Appendix C, we present numerical evidence substantiating the adequacy of this phase-field version of the EC conditions. To provide a simple heuristic argument to support it, we consider a straight smeared crack along the $x_{1}$ direction. The corresponding profiles of the fields $u$ and $\phi$ along the $x_{2}$ direction are

$$
\begin{gathered}
u\left(x_{2}\right)= \begin{cases}u^{+} & x_{2}>\alpha_{\kappa}, \\
\frac{u^{+}-u^{-}}{2 \alpha_{\kappa}} x_{2}+\frac{u^{+}+u^{-}}{2} & -\alpha_{\kappa} \leq x_{2} \leq \alpha_{\kappa}, \\
u^{-} & x_{2}<-\alpha_{\kappa},\end{cases} \\
\phi\left(x_{2}\right)= \begin{cases}\phi^{+} & x_{2}>\alpha_{\kappa}, \\
\frac{\phi^{+}-\phi^{-}}{2 \alpha_{\kappa}} x_{2}+\frac{\phi^{+}+\phi^{-}}{2} & -\alpha_{\kappa} \leq x_{2} \leq \alpha_{\kappa}, \\
\phi^{-} & x_{2}<-\alpha_{\kappa},\end{cases}
\end{gathered}
$$




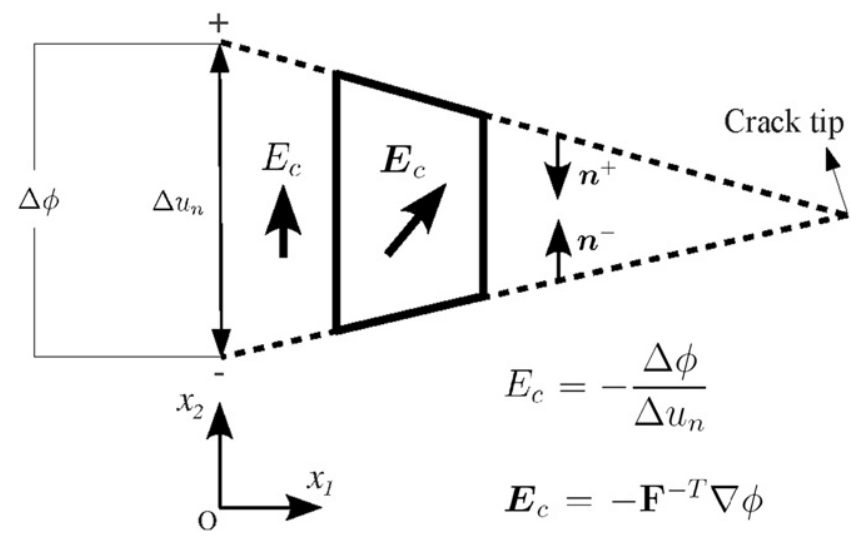

Fig. 2. Configuration of an opening crack where the electric fields $E_{c}$ and $\boldsymbol{E}_{c}$ are presented for the sharp and diffuse crack models, respectively. The crack faces are indicated by the dashed lines and a portion of the fractured zone is presented by the solid lines.

while the profile of the field $v$ is given in Eq. (2) with $d(x)=\left|x_{2}\right|$. According to the second term of Eq. (24), the electrical enthalpy of the smeared crack can then be written as

$$
H_{c}=-\frac{\varepsilon_{0}}{2} \int_{S_{c}} \int_{-\alpha_{\kappa}}^{\alpha_{\kappa}}\left|\mathbf{F}^{-T} \nabla \phi\right|^{2} J \mathrm{~d} x_{2} \mathrm{~d} S=-\frac{\varepsilon_{0}}{2} \int_{S_{c}} 2 \alpha_{\kappa}\left|\mathbf{F}^{-T} \nabla \phi\right|^{2} J \mathrm{~d} S=-\frac{\varepsilon_{0}}{2} \int_{S_{c}} \frac{\left(\phi^{+}-\phi^{-}\right)^{2}}{2 \alpha_{\kappa}+u^{+}-u^{-}} \mathrm{d} S,
$$

where we have used

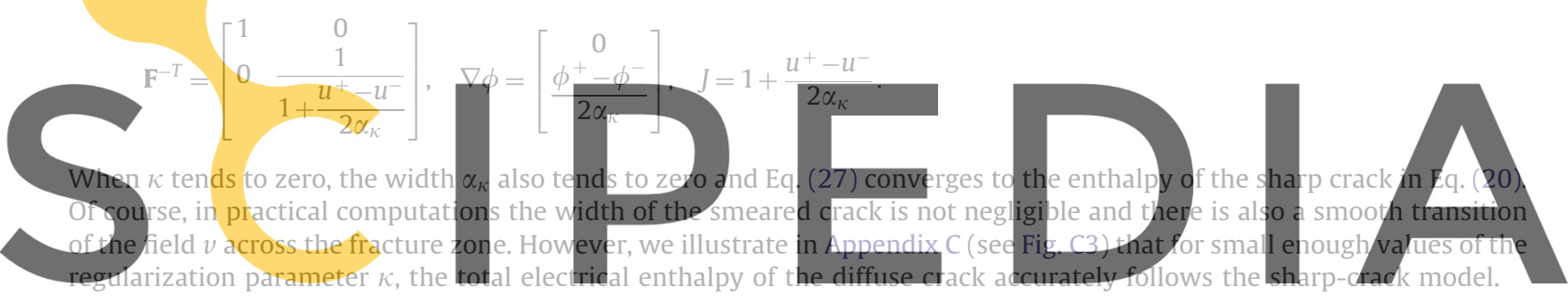

The first term in Eq. (24) is the enthalpy term of the impermeable crack model. Therefore this equation indicates that

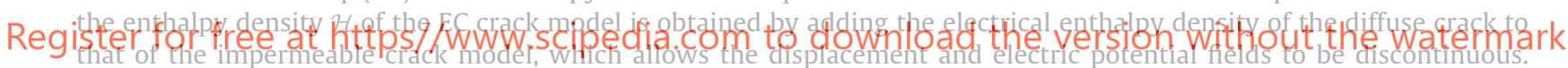

Given the enthalpy density in the second term of Eqs. (24) and (6), the stresses and electric displacements are obtained in the fracture zone as

$$
\begin{aligned}
& \boldsymbol{\sigma}=\frac{J}{2}\left(1-v^{2}\right) \boldsymbol{\sigma}_{c}, \\
& \boldsymbol{D}=J\left(1-v^{2}\right) \mathbf{F}^{-1} \boldsymbol{D}_{c},
\end{aligned}
$$

with

$$
\begin{aligned}
& \boldsymbol{D}_{c}=\varepsilon_{0} \boldsymbol{E}_{c}=\varepsilon_{0} \mathbf{F}^{-T} \boldsymbol{E}, \\
& \boldsymbol{\sigma}_{c}=\mathbf{F}^{-1}\left(\boldsymbol{D}_{c} \otimes \boldsymbol{E}_{c}\right)+\left(\boldsymbol{D}_{c} \otimes \boldsymbol{E}_{c}\right) \mathbf{F}^{-T}-\frac{\varepsilon_{0}}{2}\left|\boldsymbol{E}_{c}\right|^{2}\left(\mathbf{F}^{-1}+\mathbf{F}^{-T}\right) .
\end{aligned}
$$

Note that the lower permittivity of the free space as compared to that of the material induces a high electric field inside the fracture zone, especially in a specimen under applied electrical loading. The free space can sustain a certain level of electric field, called dielectric strength, above which the free space experiences failure of its insulating properties, i.e. electrical breakdown. This effect can be modeled mathematically as a bound constraint on the magnitude of the electric field inside the fracture gap

$$
\left|\boldsymbol{E}_{c}\right|= \begin{cases}\left|\mathbf{F}^{-T} \nabla \phi\right| & \text { if }\left|\boldsymbol{E}_{c}\right| \leq E_{d}, \\ E_{d} & \text { if }\left|\boldsymbol{E}_{c}\right|>E_{d}\end{cases}
$$

where $E_{d}$ is the dielectric strength or the electrical discharge level of the free space. This constraint can be added to the governing equations. The corresponding Lagrange multiplier modifies the electric displacement inside the fracture zone. See Appendix D for details. We evaluate the effect of the electrical breakdown constraint in Section 5. 
In the above formulation, the free-space is assumed to be the medium filling the crack gap. Other crack-gap filling media can be considered as well by replacing the free-space permittivity $\varepsilon_{0}$ with the corresponding value $\varepsilon_{m}$ in $H_{c}$ in Eq. (24).

\subsection{Polarization boundary conditions}

In addition to the crack-face conditions discussed in the previous sections, crack-face conditions on the polarization need to be supplied for ferroelectric materials. We discuss next the two usual choices and their phase-field implementation.

\subsubsection{Free-polarization crack-face conditions}

The free-polarization boundary conditions assume that the polarization distribution is unaffected by the presence of the crack, and hence dictated by the bulk material model. Mathematically, they are written as

$$
\nabla \mathbf{p}^{+} \cdot \boldsymbol{n}=\nabla \mathbf{p}^{-} \cdot \boldsymbol{n}=\mathbf{0} .
$$

This condition can be encoded in the phase-field model by multiplying with the jump set function $\left(v^{2}+\eta_{\kappa}\right)$ the energy terms involving the gradient of polarization, i.e. the domain wall energy density $U$.

1.3.2. Zero-polarization crack-face conditions

In contrast to the bulk material, the polarization vanishes in the free space filling the crack gap. The zero-polarization boundary conditions assume that the polarization is continuous at the material-free space interface, i.e.

$$
\mathbf{p}^{+}=\mathbf{p}^{-}=\mathbf{0} \text {. }
$$

This condition can also be formulated in a similar way, by multiplying with the jump set function the energy terms associated with the polarization. In Appendix C (see Fig. C4), we show that these methods indeed produce numerical solutions satisfying the free- and zero-polarization boundary conditions in a diffuse sense.

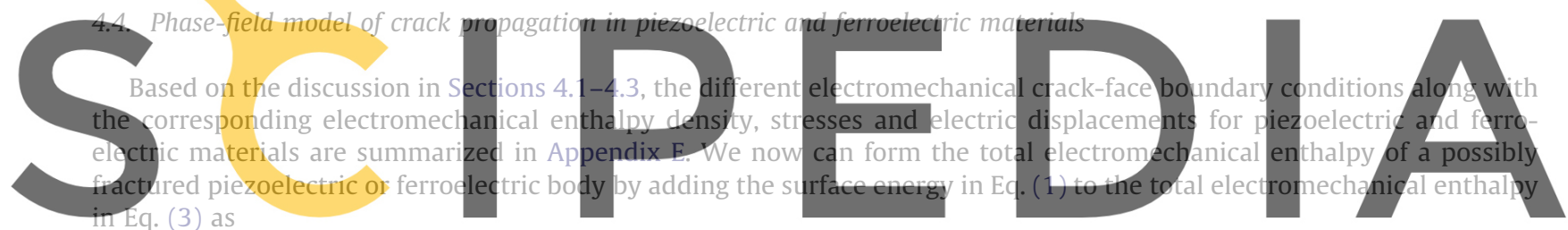

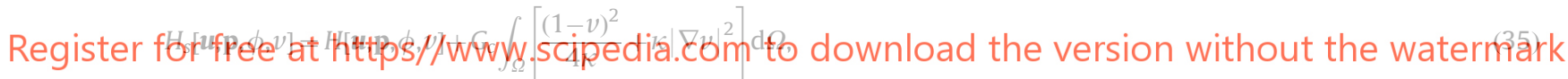

where $H$ now depends also on the field $v$ through the modified electromechanical enthalpy density $\mathcal{H}$ for different crackface boundary conditions.

To capture interactions between the fracture and the microstructure processes in ferroelectrics, the crack propagation should not be overwhelmingly faster than the microstructure evolution, and vice versa. In practice, the relative kinetics of the microstructure evolution and the crack propagation gives the two phenomena a chance to interact. In the absence of detailed experimental or theoretical information on this kinetics, $v$ is selected together with the polarization as primary order parameters and finite mobilities are introduced for the fracture and microstructure processes. Then, the time evolution of the system results from the gradient flow of the primary order parameters, assuming that the displacement and the electric field adjust immediately to mechanical and electrostatic equilibrium. The gradient flow of the polarization and the weak form of the mechanical and electrostatic equilibrium have already been introduced in Eqs. (10) and (9), respectively. Here, the gradient flow of the field $v$ as an additional governing equation is obtained as

$$
\mu_{v} \int_{\Omega} \dot{v} \delta v \mathrm{~d} \Omega=-\delta H_{s}[\boldsymbol{u}, \mathbf{p}, \phi, v ; \delta v]=-\int_{\Omega} \frac{\partial \mathcal{H}}{\partial v} \delta v \mathrm{~d} \Omega-G_{c} \int_{\Omega}\left(\frac{v-1}{4 \kappa} \delta v+\kappa v_{, i} \delta v_{, i}\right) \mathrm{d} \Omega,
$$

where $1 / \mu_{v}$ is the mobility of the fracture process. In fact this equation is a Ginzburg-Landau type evolution equation, where the left-hand side term is the dissipation in the process zone and the mobility constant controls the rate of the energy dissipation (Hakim and Karma, 2009). A recent study, using a phase-field model of brittle fracture, indicated that the mobility parameter controls the crack velocity, particularly at the initial stages of an unstable crack propagation (Kuhn and Müller, 2010). Therefore the proper selection of this parameter allows us to track crack propagation, even in mechanically unstable conditions.

The finite element equations are obtained from the weak forms in Eqs. (10), (9), and (36) with the enthalpy density $\mathcal{H}$, the stresses $\boldsymbol{\sigma}$, and the electric displacements $\boldsymbol{D}$ for each crack face condition given in Appendix E. Eqs. (10) and (36) are discretized in time with an implicit scheme from time $t_{m-1}$ to $t_{m}=t_{m-1}+\Delta t$. The Newton-Raphson method is implemented for the implicit time integration of Eq. (10) due to the nonlinear terms of the phase-separation energy $(\chi)$. Algorithm 1 presents a simple procedure to solve forward in time the coupled system in a staggered, iterative way. The 
function $\mathbf{g}$ encodes the data for the applied electromechanical load as a function of the time step. Since the crack should not be allowed to heal (irreversibility condition), the nodes reaching a value of $v$ below a certain threshold $\gamma$, are assigned a fixed value of $v=0$ for the rest of the calculation. Note that the piezoelectric models do not require the polarization data and the polarization evolution in step 6 of the algorithm is only computed for ferroelectrics. In this step the constraint for the polarization evolution is only used for the zero-polarization conditions. In fact, with this constraint the mobility term of the gradient flow is fixed to zero in the fractured zone $(v=0)$.

Algorithm 1. For crack propagation in piezoelectric and ferroelectric materials.

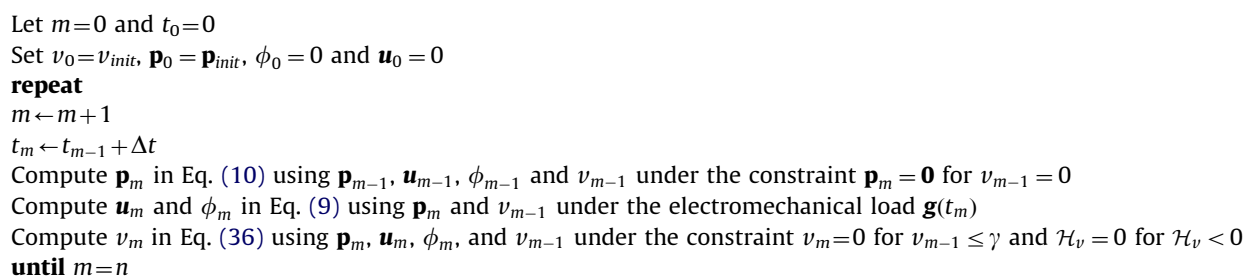

We note that the EC conditions discussed in Section 4.2 introduce non-linear terms into the finite element equations. Here, we implement an internal loop in step 7 of Algorithm 1 to solve this non-linearity. In each iteration of this loop, the nonlinear terms are updated with the last values of the mechanical displacement and the electric potential, then Eq. (9) is solved. This iteration continues until a steady state is reached for both the mechanical displacement and the electric potential. An important aspect of electromechanical crack propagation deserves special attention. Generically, the enthalpy functional can be written as

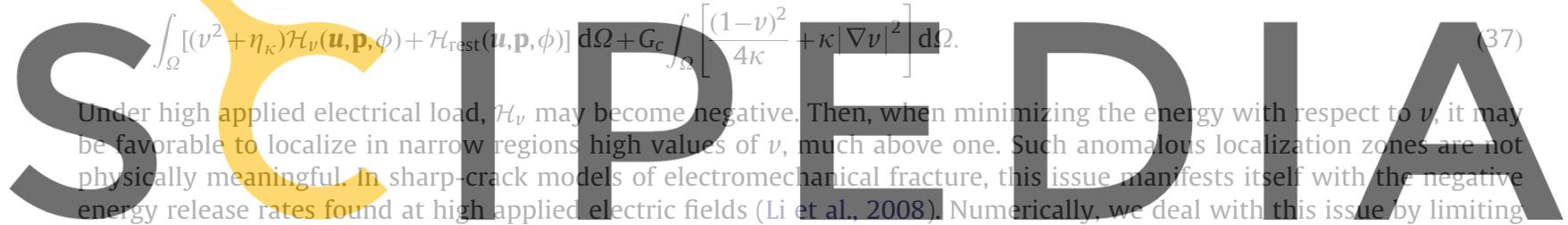

the maximum value of $v$ to one, or alternatively, by setting negative values of $\mathcal{H}_{v}$ to zero in step 8 of Algorithm 1.

Register for free at https/fyww.scipedia.com to download the version without the watermark

In this section, we examine the effects of the different crack-face conditions on the crack propagation. The simulations of a propagating crack in a poled piezoelectric material under different applied electromechanical loadings are presented in Section 5.1. We also exercise the model with different crack-gap filling dielectric fluids and introducing the constraint of electrical breakdown as discussed in Section 4.2. Section 5.2 presents the results of crack propagation in a poled ferroelectric single crystal where the crack interacts with domain switching. The results of each section are discussed in detail.

\subsection{Propagating cracks in piezoelectrics}

To investigate the effects of the different crack-face conditions on the crack propagation, a set of simulations is performed following Algorithm 1. A four-point bending setup is considered. Fig. 3 presents the dimensions of the specimen along with the boundary conditions and the pre-crack. Plane strain conditions and the material properties of PZT-PIC 151 poled along the $x_{2}$-axis (normal to the pre-crack) are assumed (see Appendix B for the material parameters). The value of $a$ is the length of the region with $v=0$ (i.e. the crack length). The pre-crack with a length of $a=0.5 \mathrm{~mm}$ is introduced in the model using the profile in Eq. (2). The model is discretized with a fine mesh, with the smallest element size of $h=10^{-4}$ mm in the vicinity of the pre-crack. An adaptive mesh refinement algorithm is employed to generate the mesh. A typical mesh is presented in Fig. 4. The regularization parameter $\kappa$ is selected as four times the smallest element size, i.e. $\kappa=4 \times 10^{-4} \mathrm{~mm}$, and the residual stiffness is set to $\eta=10^{-6}$. The inverse mobility of the fracture evolution is set to $\mu_{v}=0.1 \mathrm{Ns} / \mathrm{m}^{2}$. The point load $P$ is applied incrementally using the load function $P(t)=A t$ with a rate of $A=100 \mathrm{~N} / \mathrm{s}$ and a time-step of $\Delta t=10^{-2} \mathrm{~s}$. A total number of $2.6 \times 10^{4}$ time steps are performed for each simulation. The crack initiates and becomes unstable when the load reaches $P=14 \mathrm{KN}$, at time $t=140 \mathrm{~s}$. From this time on, the point load is fixed and the crack length relative to the initial length $(\Delta a)$ is recorded at regular intervals, see Fig. 5 for the different crack-face conditions. The finite mobility of the $v$ field allows us to follow a slow crack propagation in this period. During the crack propagation, external electric fields $E= \pm 1 \mathrm{MV} / \mathrm{m}$ (see Fig. 3 for the sign convention) are applied from time $t=190 \mathrm{~s}$. It is apparent that the impermeable crack is completely arrested by the application of both the positive and negative electrical 


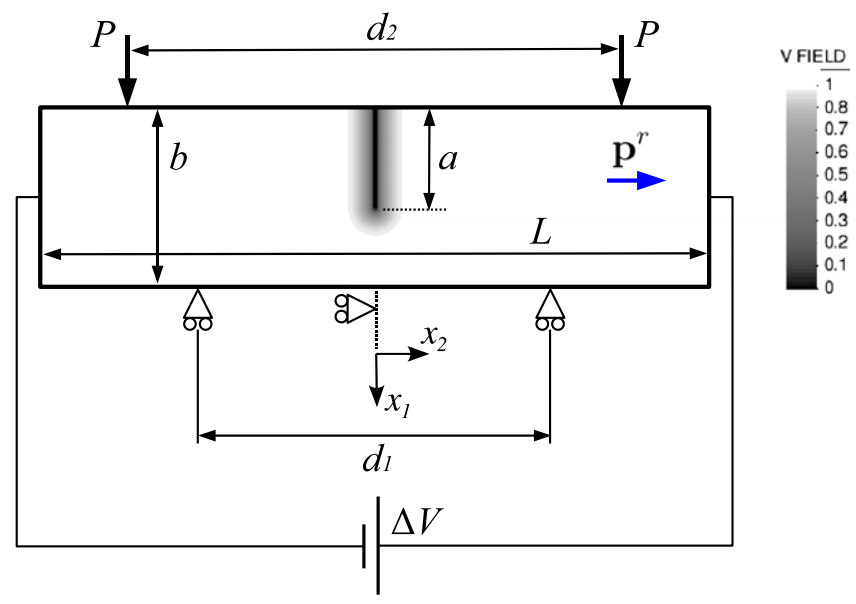

Fig. 3. Four-point bending set up with dimensions $d_{1}=10 \mathrm{~mm}, d_{2}=20 \mathrm{~mm}, L=28 \mathrm{~mm}$, and $b=4 \mathrm{~mm}$. The specimen is poled along the positive $x_{2}$ direction. Different electrical loads are applied in the $x_{2}$ direction by giving different values to $\Delta V$. The resulting applied electric field is obtained as $E=-\Delta V / L$. Note that a positive applied electric field is oriented in the poling direction.

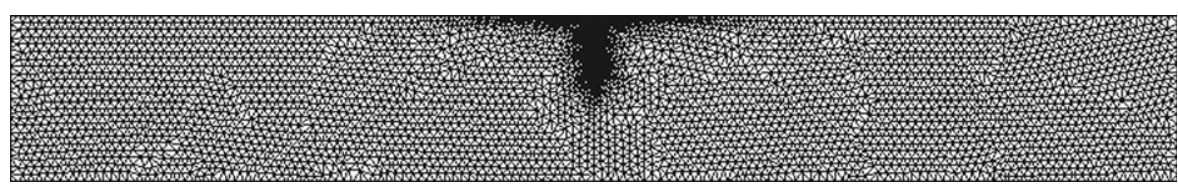

Fig. 4. Computational mesh refined near the pre-crack.

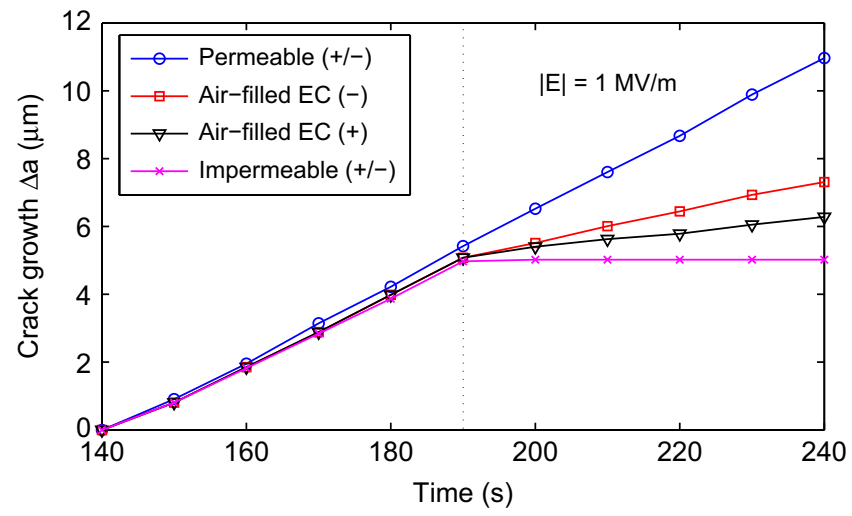

Fig. 5. Crack growth $(\Delta a)$ as a function of time in the piezoelectric material considering the permeable, impermeable and air-filled EC crack face conditions. External electric fields $E= \pm 1 \mathrm{MV} / \mathrm{m}$ are applied from time $t=190 \mathrm{~s}$. The + and - signs indicate a positive and negative applied electric field according to the sign convention given in Fig. 3.

load while the permeable crack remains unaffected by it. The crack growth rate for the EC conditions lies between that of the permeable and impermeable conditions. While the permeable and impermeable crack models show no sensitivity to the sign of the applied electric field, the air-filled EC crack is less retarded under the negative applied electric field than under the positive one. These observations, and in particular the asymmetric response of the EC crack model with respect to the sign of the applied electric field, are also apparent from the converged results of energy release rate in Fig. C1.

In the previous simulations for EC crack conditions, the crack is assumed to be filled with air with a relative permittivity of 1. To investigate the effect of the crack-gap filling fluid, analogous simulations have been carried out considering other dielectric fluids such as silicon oil and water with relative dielectric permittivities of 2.5 and 80 , respectively. Fig. 6 presents the results of the crack growth. Due to the high dielectric permittivity of water, the water-filled EC crack shows a qualitatively similar behavior to a permeable crack, i.e. it remains unaffected by the application of the external electric field. The effect of the electrical load on the crack growth is more pronounced in silicon-oil and air-filled cracks due to the lower permittivities of these media. However, if the constraint of electrical breakdown is applied as discussed in Section 4.2, the crack growth becomes less affected by the electrical load. This is apparent from the graph of air-filled crack with a dielectric strength of $E_{d}=500 \mathrm{MV} / \mathrm{m}$. By decreasing the value of dielectric strength to $E_{d}=1 \mathrm{MV} / \mathrm{m}$, of the order of the experimentally 


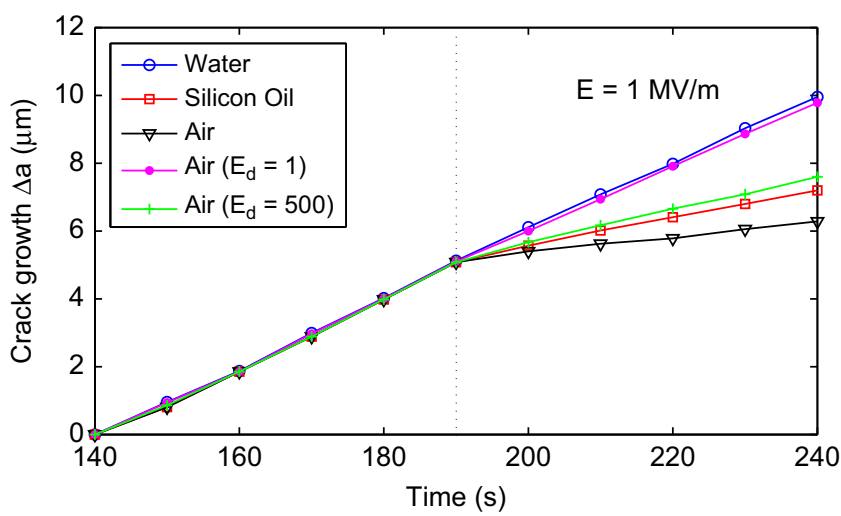

Fig. 6. Crack growth $(\Delta a)$ as a function of time in the piezoelectric material for EC crack-face conditions. The graphs correspond to water, silicon oil and airfilled crack gaps, without the electrical breakdown constraint. Two additional graphs are shown for the air-filled crack with the electrical breakdown constraint corresponding to two different values of the dielectric strength $E_{d}$. An external electric field $E=1 \mathrm{MV} / \mathrm{m}$ is applied from time $t=190 \mathrm{~s}$.

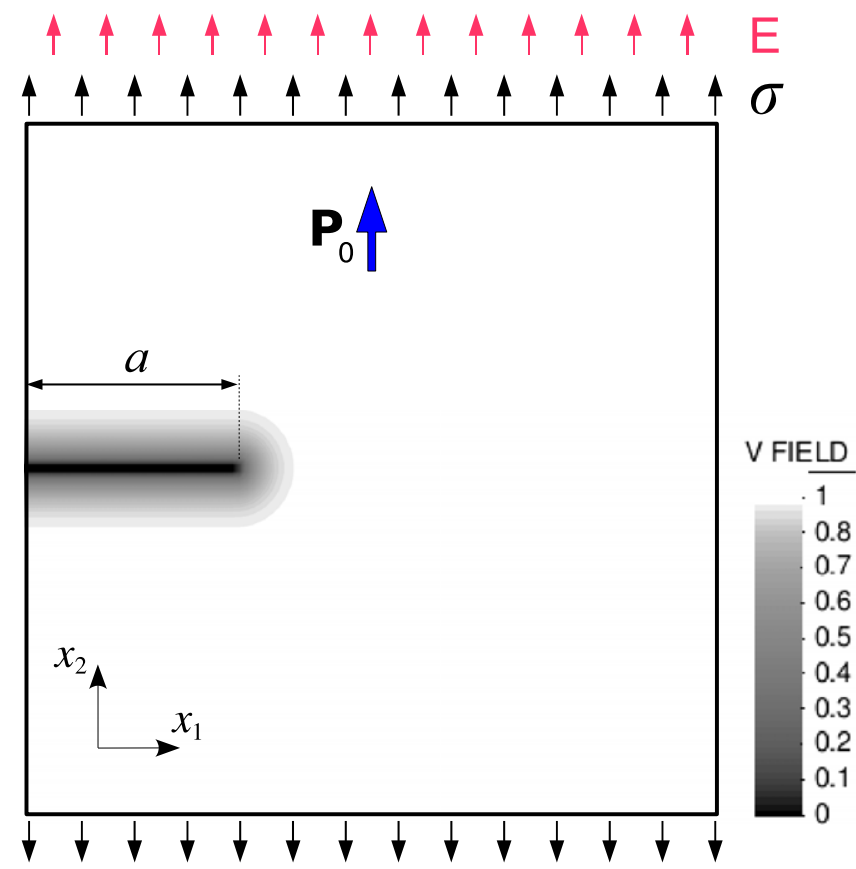

Fig. 7. A schematic of the ferroelectric computational model. The initial polarization $\mathbf{p}_{0}$ is along the $x_{2}$-axis, perpendicular to the pre-crack. The upper and bottom surfaces are subjected to a uniform tensile stress of mode I and an electric field is applied parallel to the initial polarization. An applied positive electric field is oriented in the direction of the initial polarization. The normalized dimensions of the domain are $200 \times 200$ and the normalized length of the pre-crack is $a=80$.

measured value for air (Tipler, 1987), the crack behavior approaches that of a permeable crack. Interestingly, experiments also confirm that the effective permittivity inside the air-filled crack is much higher than that of air and the effect of applied electric fields in retarding crack growth decreases significantly with increasing the permittivity of the crack interior (Schneider et al., 2003; Engert et al., 2011).

\subsection{Propagating cracks in ferroelectrics}

For the simulations of crack propagation in ferroelectric materials, we consider a rectangular domain with the loading, the boundary conditions and the pre-crack presented in Fig. 7. For convenience, dimensionless variables are selected, see Appendix A. The material parameters are chosen to fit the behavior of single crystals of barium titanate $\left(\mathrm{BaTiO}_{3}\right)$. The domain is discretized such that smaller elements are located near the pre-crack. A fine mesh with $h=0.1$ and $\kappa=0.4$ is selected. The upper and bottom surfaces are subjected to a uniform normalized tensile stress $\sigma_{22}{ }^{\prime}=0.7$. The initial polarization $\mathbf{p}_{0}$ is aligned with the $x_{2}$-axis, perpendicular to the pre-crack. We set $\phi$ to zero in the upper and lower boundaries $(E=0)$, and 
traction free, open circuited $(\boldsymbol{D} \cdot \boldsymbol{n}=0)$ conditions on the lateral faces. We consider free-polarization conditions in all the boundary of the rectangular domain. The inverse mobility of the polarization evolution is also set to $\mu_{p}=5 \times 10^{-2}$.

First, as mentioned also in the verification simulations in Appendix $C$, the initial polarization should be relaxed around the fixed pre-crack. This is done by fixing the field $v$ and executing Algorithm 1 without solving Eq. (36). The relaxation of the initial polarization $\mathbf{p}_{0}$ requires $5 \times 10^{3}$ time steps of normalized length $\Delta t^{\prime}=10^{-2}$. This time-step has been checked to provide converged and accurate solutions for the time integration of the gradient flow in Eq. (10).

After this initial relaxation, the simulations proceed solving for the fracture evolution, Eq. (36), as well. During these time steps, the crack propagation interacts with the ferroelectric domains. The same normalized time step is also considered for the time integration of Eq. (36). The threshold to detect the irreversibly fractured regions is set to $\gamma=2 \times 10^{-2}$ and the inverse mobility of the fracture evolution to $\mu_{v}=5$. The free-polarization models, summarized in Table E3, are considered for

a

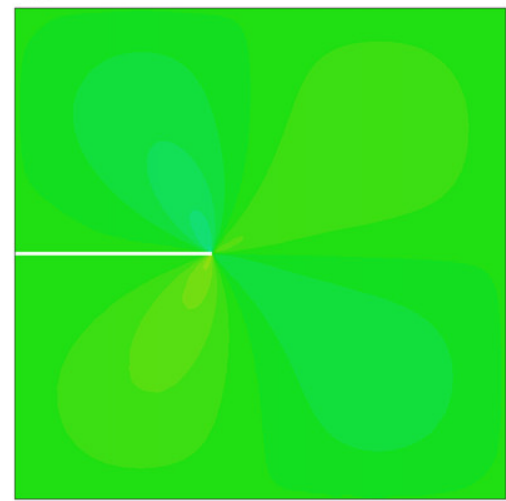

b

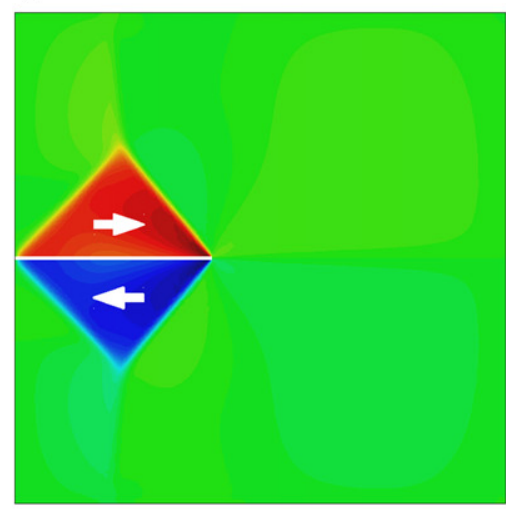

C

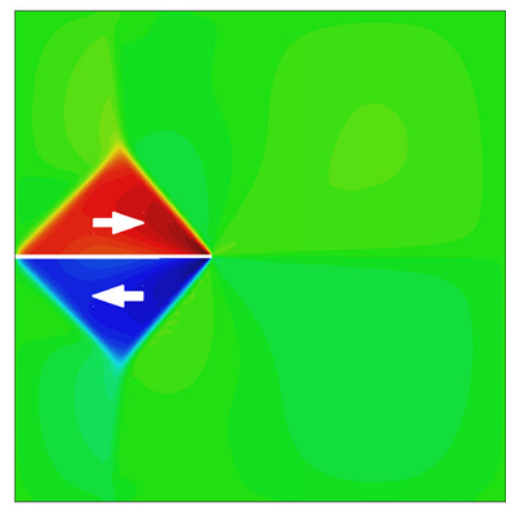

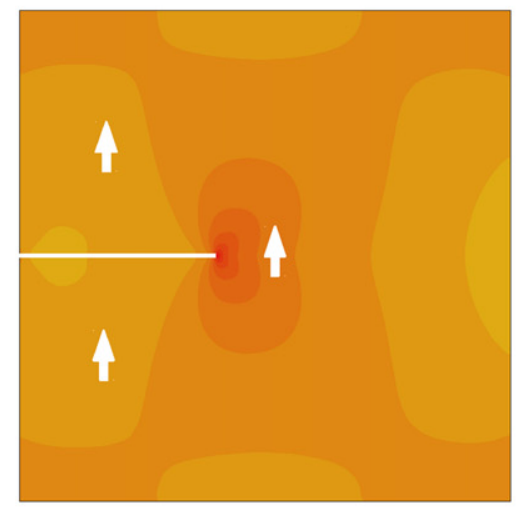
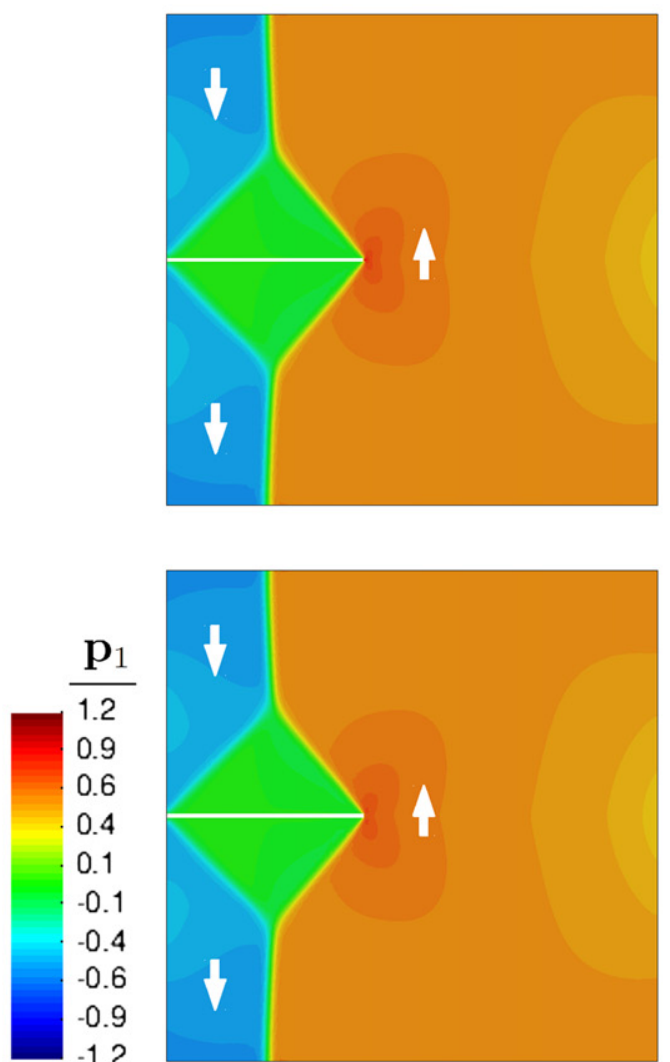

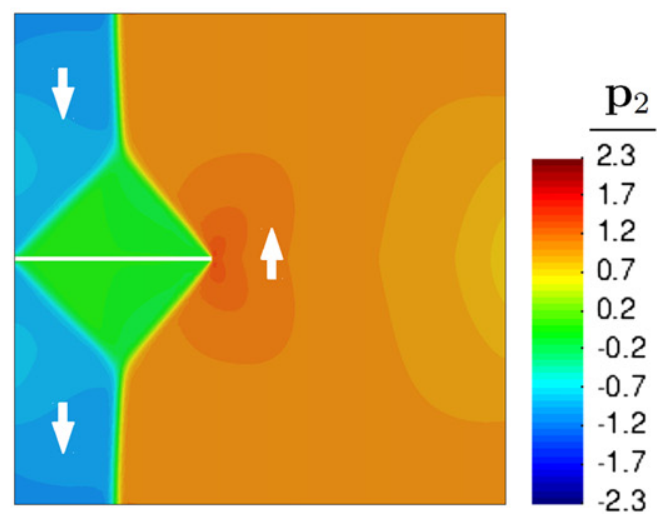

Fig. 8. Distribution of the polarization field in the ferroelectric sample under just mechanical loading (no applied electric field) for the different crack face conditions (a) permeable, (b) air-filled EC and (c) impermeable. These snapshots correspond to crack initiation instants. The left and right columns show the $x_{1}$ and $x_{2}$ components of polarization, respectively. Domain orientations are indicated with arrows. The nodes with $v=0$ are plotted in white to show the crack position. 
the simulations. Fig. 8 presents snapshots of the ferroelectric domain formation around the cracks for the different crack-face conditions under mechanical load, namely: (a) traction-free, electrically permeable (permeable in short), (b) air-filled energetically consistent (EC), and (c) traction-free, electrically impermeable (impermeable in short). These snapshots are taken at the instant when the cracks propagation initiates. Permeable cracks have the weakest influence on the polarization fields as shown in Fig. 8(a). The polarization intensity increases near the crack tip due to the concentration of stresses, but only a weak ferroelastic switching forming wing-shaped domains is apparent in Fig. 8(a, left), and more clearly in Fig. 9. Since the stresses are applied parallel to the polarization direction, the mechanical load cannot cause any additional switching and $90^{\circ}$ ferroelastic domain switching becomes unfavorable. The prominent effect arising from the permeable conditions is the elongation of the material in front of the crack tip perpendicularly to the crack. On the other hand, air-filled EC and impermeable cracks have a significant influence on the polarization fields as shown in Fig. 8(b) and (c), respectively. Both crack conditions force the polarization to rotate $90^{\circ}$ and to form two triangular horizontally polarized domains around the fracture zone. This switching is weaker for EC cracks, especially near the crack tip where the permittivity of the crack gap allows for a small penetration of the polarization. Due to this effect, the $x_{2}$ component of the polarization in front of the crack tip is larger for EC conditions with respect to impermeable conditions. Despite this small difference, the overall behavior of the air-filled EC cracks (without the electrical breakdown constraint) is quite similar to the impermeable one under just mechanical loading (recall that no external electric field is applied here). According to the results in Section 5.1 (see Fig. 5 ), one can expect the behavior of the air-filled EC crack to deviate from that of the impermeable crack under a positive (in the direction of the initial polarization) or negative (opposite to the initial polarization) electric field.

To investigate this point we have also performed simulations under both mechanical and electrical loads. A nominal electric field $E=-V / L$ is induced in the sample by setting the value of $V, L$ being the height of the model. The application of positive electric fields for all the crack-face conditions leads to the increase of the polarization in the vertical $x_{2}$ direction in front of the crack tip. A snapshot of domain formation around the air-filled EC crack under the normalized electric field $E=10^{-2}$ is presented in Fig. 10(a) at the crack initiation instant. Another effect of the positive fields is the shrinkage of $90^{\circ}$ domains with a decrease in the magnitude of the $x_{1}$ component of the polarization around the fracture zone for EC and impermeable conditions. In contrast, negative electric fields of magnitudes below the normalized coercive field $E_{c}=-8 \times 10^{-3}$ decrease the intensity of the vertical polarization and enlarge the $90^{\circ}$ domains. Fig. 10 (b) shows the domain formation for the air-filled EC crack under the normalized electric field $E=-5 \times 10^{-3}$ at the crack initiation instant. The negative field induces a $180^{\circ}$ domain switching with a reversed polarization in front of the crack. A similar switching is also observed for impermeable conditions (not shown). Above the magnitude of the normalized coercive field, the polarization reverses in the whole domain for permeable conditions. In contrast, due to the presence of the crack gap, polarization reversal occurs only in an area in front of the air-filled EC and impermeable cracks. This polarization reversal is shown in Fig. 10(c) for the air-filled EC crack under a normalized electric field $E=-10^{-2}$ at the crack initiation instant.

Analogous simulations are performed considering the zero-polarization crack models. Fig. 11 presents the distribution of the polarization magnitude for the zero- and free-polarization crack models under just mechanical loading and considering the impermeable conditions. The zero-polarization model shows a similar domain formation to that of the free-polarization model. This similarity is also observed in the simulations of the permeable and air-filled EC crack conditions and under different electromechanical loads (not shown for the sake of brevity). The main difference is that the polarization components tend to zero when approaching the fractured zone in the zero-polarization crack models, cf. Fig. 11(a) and (b). In the light of these results, it can be concluded that the electrical crack-face conditions have a significantly stronger effect on the ferroelectric domain structures around the cracks than the polarization conditions.

Finally, the effect of domain switching in the vicinity of the crack on the crack propagation is evaluated by recording the crack growth $(\Delta a)$ after 500 time steps at time $t^{\prime}=5$. Note that the applied stress $\sigma_{22}^{\prime}=0.7$ supplies enough energy for the

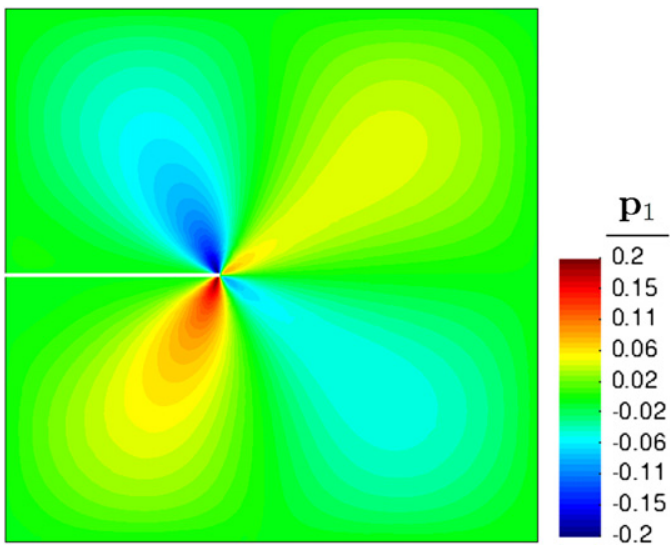

Fig. 9. Distribution of the horizontal polarization $\mathbf{p}_{1}$ around the permeable crack in the ferroelectric sample (detailed view of Fig. 8 (a, left)). The nodes with $v=0$ are plotted in white to show the crack position. 
a

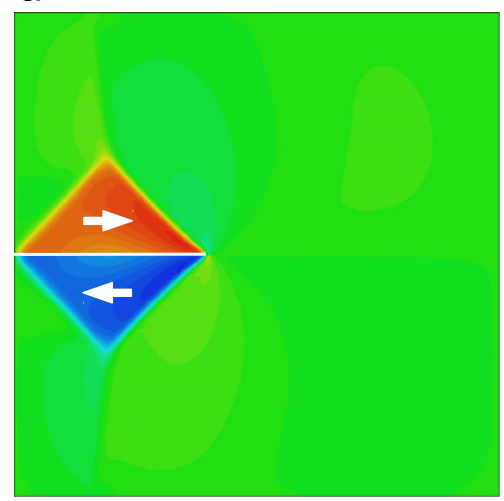

b

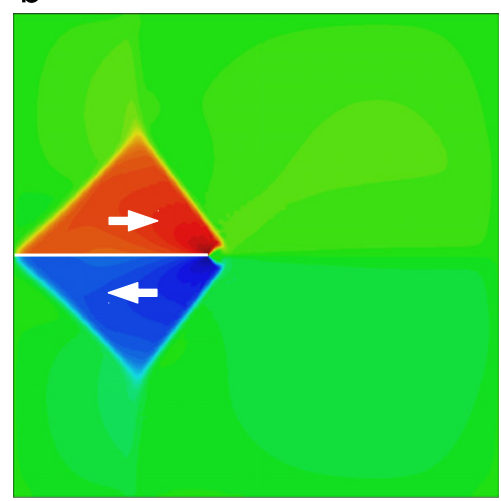

C

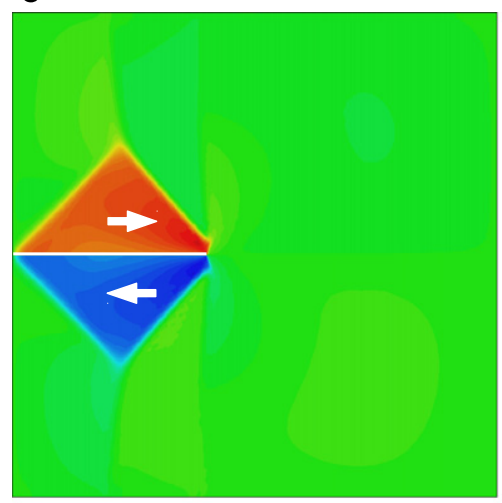

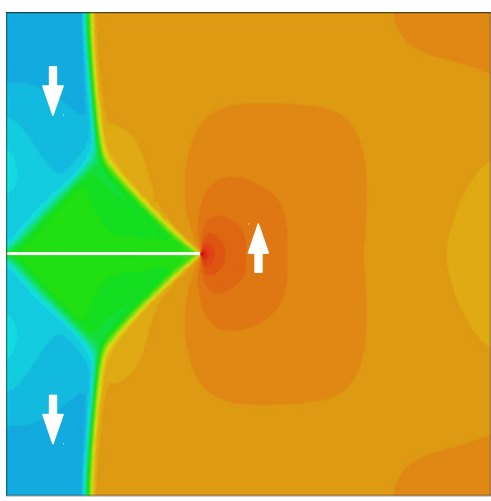
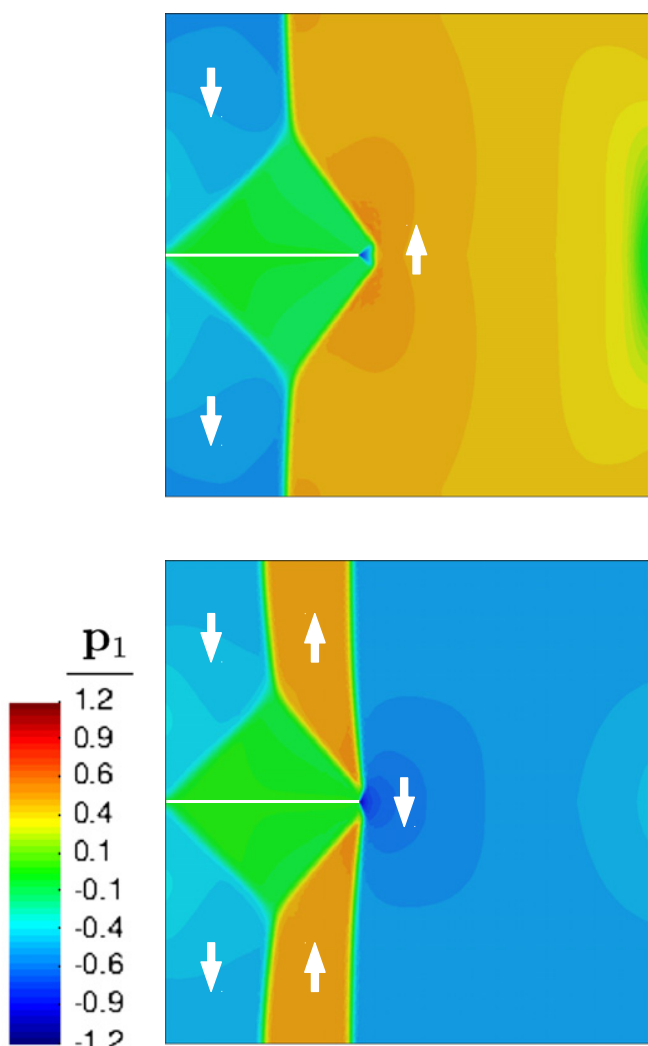

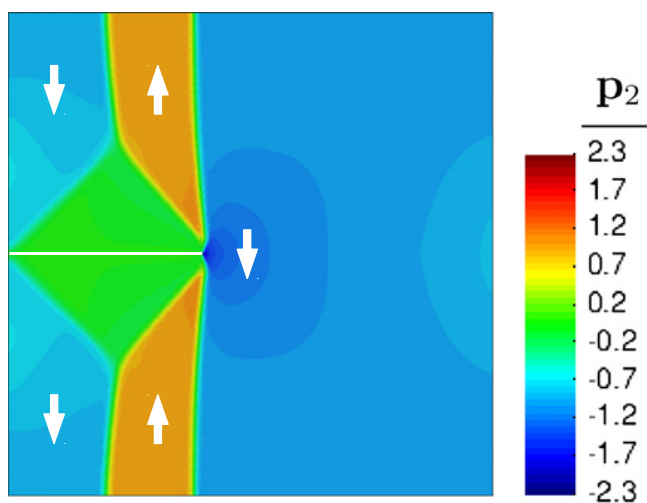

Fig. 10. Distribution of the polarization field in the ferroelectric sample for the air-filled EC crack-face conditions. The results are obtained considering different normalized applied electric fields (a) $E=10^{-2}$, (b) $E=-5 \times 10^{-3}$, (c) $E=-10^{-2}$. These snapshots correspond to the crack initiation instants, at time $t^{\prime}=1.3, t^{\prime}=0.27$, and $t^{\prime}=0.6$, respectively. The left and right columns show the $x_{1}$ and $x_{2}$ components of polarization, respectively. Domain orientations are indicated with white arrows. The points where $v=0$ are represented in white to show the crack position.

crack initiation followed by an unstable crack propagation in all the simulations. The results are presented in Fig. 12 for the different crack-face conditions and under various applied electric fields. It is obvious that the crack growth increases with the application of the negative normalized electric field $E=-5 \times 10^{-3}$ for all the crack conditions. This weakening effect is more pronounced for the EC crack model. The polarization reversal under the negative normalized electric field $E=-10^{-2}$ (above the coercive field in magnitude) decreases the crack growth significantly and shows a similar toughening effect to that of the positive applied electric fields. Similarly to piezoelectric materials, the strongest retarding effect on the crack propagation is observed for the impermeable conditions. According to Fig. 12, the impermeable crack is almost arrested under the normalized electric field $E=10^{-2}$.

All the above simulations have also been carried out with the constraint of electrical breakdown and considering different dielectric fluids filling the crack gap (water and silicon-oil), not shown. As expected, and analogously to the results reported in Section 5.1 for piezoelectric materials, the behavior of the EC model approaches that of the permeable 
a

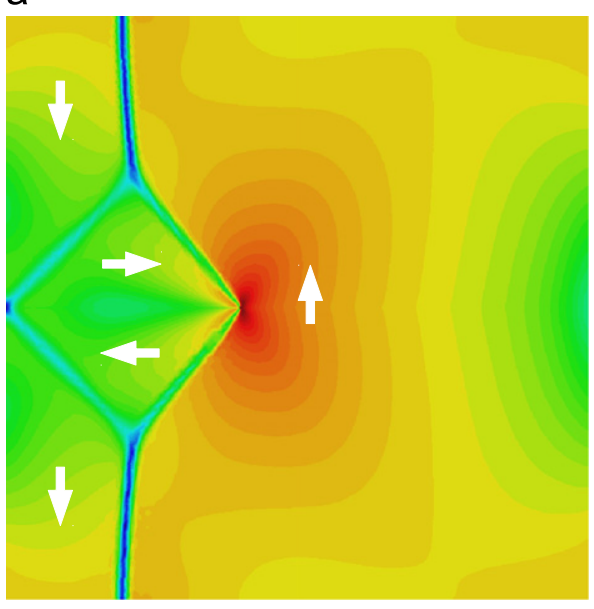

b

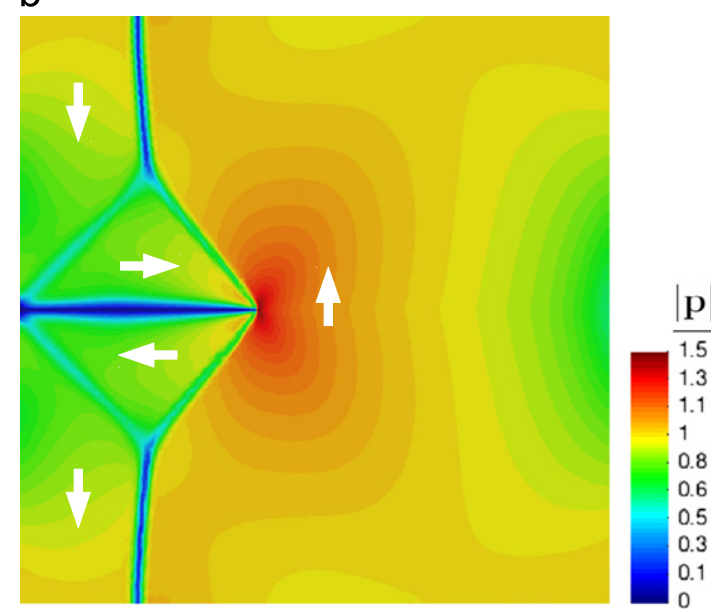

Fig. 11. Distribution of polarization magnitude $|\mathbf{p}|$ in the ferroelectric sample considering the (a) free-polarization and (b) zero-polarization models. These snapshots correspond to the crack initiation instants. Traction-free, electrically impermeable crack conditions are considered for both models. Domain orientations are indicated with white arrows.

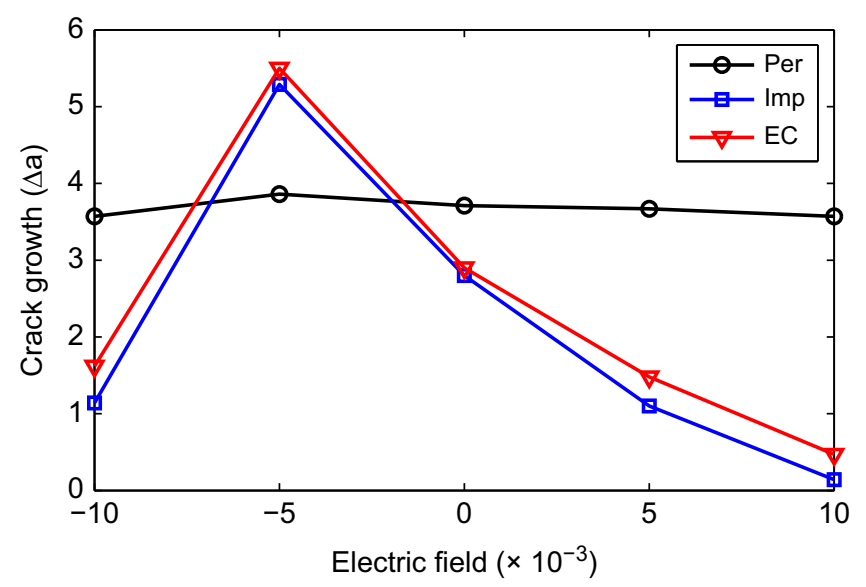

Fig. 12. Crack growth $(\Delta a)$ as a function of the magnitude and sign of the electric field in the ferroelectric material at time $t^{\prime}=5$. The results are obtained for the different crack face conditions: Permeable (Per), Impermeable (Imp) and air-filled Energetically consistent (EC).

one as the permittivity of the crack-gap filling medium increases and the value of the electric field inside the crack gap is limited to the dielectric strength. Furthermore, the simulations of the zero-polarization models (not shown) result in the similar weakening and toughening effects of the electrical loads as in the free-polarization models.

It is noteworthy that the experimental results by Ricoeur and Kuna (2003) show a retarding effect of a positive electric field on the crack propagation in $\mathrm{BaTiO}_{3}$. This retarding effect is also observed in other experiments in PZT (Wang and Singh, 1997; Shindo et al., 2002) and PMN-PT (Jiang et al., 2009). The weakening effect associated with the application of negative electric fields has also been reported in experiment (Wang and Singh, 1997; Shindo et al., 2002; Fu and Zhang, 2000; Jiang et al., 2009). The polarization reversal effect of an applied electrical load above the coercive field has been reported by Ricoeur and Kuna (2003). All of these observations are in agreement with our simulation results presented above.

A number of phase-field simulations have been performed near stationary sharp cracks, showing similar effects of the electrical loading on the fracture behavior and similar domain patterns as those in Fig. 8(c) around impermeable cracks in ferroelectric single crystals (Song et al., 2007; Xu et al., 2010b; Wang and Kamlah, 2010). The results reported by Li and Landis (2011) indicate positive energy release rates for a sharp crack with a modified form of impermeable boundary conditions under purely electrical loading, which is in agreement with the weakening effect of negative electric fields observed in our simulations. However, we have found a disagreement with the results of Wang and Zhang (2007). For permeable conditions, this paper reports that a positive electric field reduces the apparent fracture toughness, while a negative electric field enhances it. Although they presented a similar wing-shaped domains of Fig. 9, their simulation results show the movement of these domains away from the crack tip, while in our simulations the wing-shaped pattern follows the crack tip. 


\section{Conclusions}

This paper presents a family of phase-field models of fracture in piezoelectric and ferroelectric materials. These models are based on a variational formulation of brittle fracture coupled with the linear theory of piezoelectricity and a phasefield model of ferroelectric domain evolution, respectively. Different electromechanical crack conditions (defined as crackface boundary conditions in sharp-crack models) are encoded into the phase-field framework, namely (1) traction-free, electrically permeable (permeable in short), (2) traction-free, electrically impermeable (impermeable in short), and (3) energetically consistent (EC), with or without the electrical breakdown constraint, and with different crack-gap filling media. A set of simulations is performed to verify the proposed phase-field models against the corresponding sharp-crack models. The results provide numerical evidence that for small enough values of the regularization parameter $\kappa$, the proposed models recover the solutions of the sharp-crack models near the edges of the diffuse cracks. We have also conducted simulations to examine the effects of the different crack-face conditions, electromechanical loadings and crackgap filling media on the crack propagation and the microstructure evolution of the material. The main results are outlined as follows:

1. The strongest retarding effect on the crack propagation under electrical loads is observed for impermeable crack conditions. Furthermore, the crack growth rate for the EC conditions lies between that of the permeable and impermeable conditions.

2. The behavior of the EC model approaches that of the permeable one as the permittivity of the crack-gap filling medium increases and the value of the electric field inside the crack gap is limited to the dielectric strength. In particular:

- A water-filled crack shows a similar behavior to a permeable crack due to the high dielectric permittivity of water. The effect of electrical loads on the crack growth is more pronounced in silicon-oil and air-filled cracks due to the lower permittivities of these media.

O An air-filled EC crack under the constraint of electrical breakdown behaves similar to a permeable crack, which is in agreement with experimental observations (Schneider et al., 2003; Engert et al., 2011).

3. Impermeable and air-filled EC conditions without the constraint of electrical breakdown have a significant influence on the polarization fields around the cracks in ferroelectrics. Both crack conditions force the polarization to rotate $90^{\circ}$ and to form two triangular horizontally polarized domains around the fracture zone.

4. The electromechanical crack-face conditions have a significantly stronger effect on the ferroelectric domain structures around the cracks than the polarization crack-face conditions.

5. A negative electric field below the coercive field perpendicular to the crack enhances the crack propagation in ferroelectrics, while a positive electric field retards it, for all crack conditions. The magnitude of the enhancement effect depends on the crack conditions, being stronger for EC cracks. Experimental results show a qualitatively similar effect of electrical loads on the crack propagation in poled ferroelectrics (Ricoeur and Kuna, 2003; Wang and Singh, 1997; Shindo et al., 2002; Jiang et al., 2009).

6. A negative electric field above the coercive field perpendicular to the crack retards the crack propagation in ferroelectrics for all crack conditions, due to the polarization reversal in front of the crack, as has also been observed in experiment (Ricoeur and Kuna, 2003). The magnitude of the retarding effect depends on the crack conditions, being stronger for impermeable cracks.

The results reported here show the ability of our phase-field approach to elucidate the fracture behavior of ferroelectric ceramics, strongly dependent upon the specific crack conditions. However, we also suggests that more work is needed to produce predictive simulations of such complex phenomena. In particular, we have provided numerical evidence that the phase-field solutions converge to those of the corresponding sharp-crack models for small enough values of the fracture regularization parameter and the mesh size. However, a rigorous mathematical analysis would be desirable to prove this convergence. Also, the intrinsic anisotropy of the crystal, i.e. the different surface energy in different cleavage planes, is bound to have an effect on the fracture response as well. Another important issue is the quantification and relative magnitude of the parameters $\mu_{p}$ and $\mu_{v}$, which can have an important effect on the resulting response. Finally, three dimensional simulations and an extension to polycrystalline materials along the lines of Abdollahi and Arias (2012) are necessary. All these topics are the subject of current work.

\section{Acknowledgements}

The authors gratefully acknowledge the support of the Ministerio de Ciencia e Innovación (DPI2010-19145 and DPI2011-26589).

\section{Appendix A. Energy functions and material constants for $\mathrm{BaTiO}_{3}$}

The energy functions in Eq. (4) are chosen following (Devonshire, 1949, 1951), adapted to a plane polarization and plane strain state (Zhang and Bhattacharya, 2005). The stiffness tensor $\mathbb{C}$ and the eigenstrain $\boldsymbol{\varepsilon}_{s}$ can be written in Voigt 
form as

$$
\begin{gathered}
\mathbb{C}=\left(\begin{array}{ccc}
c_{1} & c_{2} & 0 \\
c_{2} & c_{1} & 0 \\
0 & 0 & c_{3}
\end{array}\right), \\
\boldsymbol{\varepsilon}_{s}(\mathbf{p})=\left(\begin{array}{c}
a p_{1}^{2}-b p_{2}^{2} \\
b p_{1}^{2}-a p_{2}^{2} \\
c p_{1} p_{2}
\end{array}\right),
\end{gathered}
$$

with

$$
\begin{aligned}
& a=\frac{b_{1} c_{1}-b_{2} c_{2}}{2\left(c_{1}^{2}-c_{2}^{2}\right)}, \\
& b=\frac{b_{2} c_{1}-b_{1} c_{2}}{2\left(c_{1}^{2}-c_{2}^{2}\right)}, \\
& c=\frac{b_{3}}{c_{3}},
\end{aligned}
$$

where $c_{i}$ and $b_{i}(i=1,2,3)$ are the elastic and electromechanical coupling constants, respectively. The energy functions $U$ and $\chi$ are stated as:

$$
\begin{aligned}
& U(\nabla \mathbf{p})=\frac{a_{0}}{2}\left(p_{1,1}^{2}+p_{1,2}^{2}+p_{2,1}^{2}+p_{2,2}^{2}\right), \\
& \chi(\mathbf{p})=\frac{a_{1}}{2}\left(p_{1}^{2}+p_{2}^{2}\right)+\frac{a_{2}}{4}\left(p_{1}^{4}+p_{2}^{4}\right)+\frac{a_{3}}{2}\left(p_{1}^{2} p_{2}^{2}\right)+\frac{a_{4}}{6}\left(p_{1}^{6}+p_{2}^{6}\right)+\frac{a_{5}}{4}\left(p_{1}^{4} p_{2}^{4}\right),
\end{aligned}
$$

where $a_{0}$ is the scaling parameter of the domain wall energy and $a_{i}(i=1, \ldots, 5)$ are the constants of the phase separation potential $\chi$. This potential includes only an eight-order term as proposed by Zhang and Bhattacharya (2005) and it can be improved by adding other high order terms to reproduce the dielectric behavior of barium titanate single crystals in a more accurate way (Li et al., 2005; Wang et al., 2007). Nevertheless, note that the role of the eighth order cross-term $p_{1}^{4} p_{2}^{4}$ is the most important among all high order terms for the phase-field modeling of ferroelectric domains in the tetragonal phase. In fact, this term provides a reasonable energy barrier for $90^{\circ}$ domain switching, while allowing the other terms to fit the dielectric behavior of the material (Zhang and Bhattacharya, 2005). Furthermore, to simplify the model and the numerical solution, it is assumed that the elastic properties of the material have at most the cubic symmetry of the paraelectric phase. However, an accurate fit to the elastic properties of the tetragonal phases can be obtained by adding sixth order coupling terms to the phase-field formulation, which results in different elastic properties of the domain walls (Su and Landis, 2007).

For convenience, dimensionless variables are selected through the following normalizations: $x_{i}^{\prime}=x_{i} \sqrt{c_{0} / a_{0}} / p_{0}$, $p_{i}^{\prime}=p_{i} / p_{0}, t^{\prime}=t c_{0} / \mu p_{0}^{2}, \varepsilon_{0}^{\prime}=\varepsilon_{0} c_{0} / p_{0}^{2}, \phi^{\prime}=\phi / \sqrt{a_{0} c_{0}}, \quad a_{1}^{\prime}=a_{1} p_{0}^{2} / c_{0}, \quad a_{2}^{\prime}=a_{2} p_{0}^{4} / c_{0}, a_{3}^{\prime}=a_{3} p_{0}^{4} / c_{0}, a_{4}^{\prime}=a_{4} p_{0}^{6} / c_{0}, a_{5}^{\prime}=a_{5} p_{0}^{8} / c_{0}$, $b_{i}^{\prime}=b_{i} p_{0}^{2} / c_{0}$, and $c_{i}^{\prime}=c_{i} / c_{0}$, where $i=1,2,3$. The equations with normalized variables are the same as the original ones. The constants are chosen to fit the behavior of single crystals of barium titanate $\left(\mathrm{BaTiO}_{3}\right)$ at room temperature, taking $c_{0}=1 \mathrm{GPa}$, a value for the spontaneous polarization of $p_{0}=0.26 \mathrm{C} \mathrm{m}^{-2}$, the relative spontaneous strains $\varepsilon_{a}=-0.44 \%$ along $a$-axis and $\varepsilon_{c}=0.65 \%$ along $c$-axis (Zhang and Bhattacharya, 2005; Wang et al., 2007). The domain wall scaling parameter is set to $a_{0}=3.7 \times 10^{-9} \mathrm{Vm}^{3} \mathrm{C}^{-1}$, which leads to the value of $0.5 \mathrm{~nm}$ for the normalized unit length $\Delta x^{\prime}=1$. By setting the normalized scaling parameter of the domain wall energy to $a_{0}^{\prime}=0.1$, the normalized width of domain walls is obtained between 4 and 6 in the simulations, corresponding to $2-3 \mathrm{~nm}$, in the order of experimentally measured values in tetragonal ferroelectric ceramics (Stemmer et al., 1995; Floquet et al., 1997). The intrinsic fracture toughness of $\mathrm{BaTiO}_{3}$ is taken as $K_{c}=0.49 \mathrm{MPa} \sqrt{m}$ from experimental measurements on an annealed sample (Meschke et al., 2000). Considering Young's modulus and Poisson's ratio for $\mathrm{BaTiO}_{3}$ as $E=100 \mathrm{GPa}$ and $v=0.37$ respectively, consistent with the elastic constants $c_{i}(i=1,2,3)$, the value of the critical energy release rate in plane strain is obtained as $G_{c}=\left(1-v^{2}\right) K_{c}^{2} / E=2 \mathrm{~J} \mathrm{~m}{ }^{-2}$. The value of normalized critical energy release rate is then calculated as $G_{c}^{\prime}=G_{c} \sqrt{1 / a_{0} c_{0}} / p_{0}=4$. The normalized parameters are presented in Table A1. With the selected parameters, the normalized Landau-Devonshire energy furnishes a multi-well energy landscape with four minima corresponding to the four variants of the tetragonal phase with normalized polarization $p^{\prime}=(1,0),(0,1),(-1,0)$ and $(0,-1)$. 
Table A1

Normalized parameters.

\begin{tabular}{llllllll}
\hline$c_{1}^{\prime}$ & $c_{2}^{\prime}$ & $c_{3}^{\prime}$ & $b_{1}^{\prime}$ & $b_{2}^{\prime}$ & $b_{3}^{\prime}$ & $a_{0}^{\prime}$ & \\
\hline 185 & 111 & 74 & 1.4282 & -0.185 & 0.8066 & 0.1 \\
$c^{\prime}$ & $a_{1}^{\prime}$ & $a_{2}^{\prime}$ & $a_{3}^{\prime}$ & $a_{4}^{\prime}$ & $\varepsilon_{0}^{\prime}$ & 0.0065 \\
\hline 0.0109 & -0.007 & -0.009 & 0.018 & 0.0261 & 5 & 4 \\
\hline
\end{tabular}

\section{Appendix B. Constitutive matrices for PZT-PIC 151}

The constitutive matrices for PZT-PIC 151 can be written in Voigt form for the global coordinate system as

$$
\begin{aligned}
& \left\{\begin{array}{l}
\sigma_{11} \\
\sigma_{22} \\
\sigma_{33} \\
\sigma_{23} \\
\sigma_{13} \\
\sigma_{12}
\end{array}\right\}=\left[\begin{array}{cccccc}
c_{11} & c_{13} & c_{12} & 0 & 0 & 0 \\
c_{13} & c_{33} & c_{13} & 0 & 0 & 0 \\
c_{12} & c_{13} & c_{11} & 0 & 0 & 0 \\
0 & 0 & 0 & c_{44} & 0 & 0 \\
0 & 0 & 0 & 0 & \frac{c_{11}-c_{12}}{2} & 0 \\
0 & 0 & 0 & 0 & 0 & c_{44}
\end{array}\right]\left\{\begin{array}{l}
\varepsilon_{11} \\
\varepsilon_{22} \\
\varepsilon_{33} \\
\gamma_{23} \\
\gamma_{13} \\
\gamma_{12}
\end{array}\right\}-\left[\begin{array}{ccc}
0 & e_{31} & 0 \\
0 & e_{33} & 0 \\
0 & e_{31} & 0 \\
0 & 0 & e_{15} \\
0 & 0 & 0 \\
e_{15} & 0 & 0
\end{array}\right]\left\{\begin{array}{l}
E_{1} \\
E_{2} \\
E_{3}
\end{array}\right\} \\
& \left\{\begin{array}{l}
D_{1} \\
D_{2} \\
D_{3}
\end{array}\right\}=\left[\begin{array}{cccccc}
0 & 0 & 0 & 0 & 0 & e_{15} \\
e_{31} & e_{33} & e_{31} & 0 & 0 & 0 \\
0 & 0 & 0 & e_{15} & 0 & 0
\end{array}\right]\left\{\begin{array}{l}
\varepsilon_{11} \\
\varepsilon_{22} \\
\varepsilon_{33} \\
\gamma_{23} \\
\gamma_{13} \\
\gamma_{12}
\end{array}\right\}+\left[\begin{array}{ccc}
\kappa_{11} & 0 & 0 \\
0 & \kappa_{33} & 0 \\
0 & 0 & \kappa_{11}
\end{array}\right]\left\{\begin{array}{l}
E_{1} \\
E_{2} \\
E_{3}
\end{array}\right\} .
\end{aligned}
$$

The material is transversely isotropic with the positive $x_{2}$-axis as the poling direction and the $x_{1}-x_{3}$ plane as the isotropic plane. The material data are (Heyer et al., 1998; Li et al., 2008)

$$
\begin{aligned}
& c_{11}=110 \times 10^{9} \mathrm{~N} / \mathrm{m}^{2}, \quad c_{13}=64 \times 10^{9} \mathrm{~N} / \mathrm{m}^{2}, \quad c_{12}=63 \times 10^{9} \mathrm{~N} / \mathrm{m}^{2}, \\
& c_{33}=100 \times 10^{9} \mathrm{~N} / \mathrm{m}^{2}, \quad c_{44}=20 \times 10^{9} \mathrm{~N} / \mathrm{m}^{2}, \\
& e_{31}=-9.6 \mathrm{C} / \mathrm{m}^{2}, \quad e_{33}=15.1 \mathrm{C} / \mathrm{m}^{2}, \quad e_{15}=12 \mathrm{C} / \mathrm{m}^{2}, \\
& \kappa_{11}=98.24 \times 10^{-10} \mathrm{C} / \mathrm{Vm}, \quad \kappa_{33}=75.3 \times 10^{-10} \mathrm{C} / \mathrm{Vm} .
\end{aligned}
$$

The fracture toughness, Young's moduli, and Poisson's ratio for PZT-PIC 151 are selected as $K_{c}=1 \mathrm{MPa} \sqrt{m}, E=67 \mathrm{GPa}$ and $v=0.36$ (consistent with the elastic constants in $\mathbb{C}$ ), respectively, leading to the critical energy release rate in plane strain $G_{c}=\left(1-v^{2}\right) K_{c}^{2} / E=13 \mathrm{~J} / \mathrm{m}^{2}$.

\section{Appendix C. Verification}

In this appendix, we present a set of numerical simulations aimed at verifying different aspects of the proposed models. In all cases stationary cracks are considered for simplicity. We first study the convergence of the energy-release rate as computed with the phase-field models proposed in Section 4 for piezoelectrics. We next present numerical evidence that the phase-field solutions, both for piezoelectrics and ferroelectrics, satisfy the corresponding crack-face conditions in a diffuse sense, i.e. in the limit of vanishingly small regularization parameter $\kappa$, and converge to the solutions of the corresponding sharp-crack model as the mesh size $h$ and $\kappa$ tend to zero in a concerted manner. For the EC model, we show that the proposed electrical enthalpy of the fracture zone converges to the enthalpy of the crack gap in the corresponding sharp-crack model.

\section{C.1. Phase-field models for fracture in piezoelectrics}

As a first verification test, we compare the energy release rates in a piezoelectric specimen computed with the electromechanical enthalpies proposed in Sections 4.1 and 4.2 to those obtained with the corresponding sharp-crack model by Li et al. (2008). The energy-release rate is defined as the reduction in the potential energy of the cracked body per unit increase in the crack area (Anderson, 1991). Then the energy-release rate is obtained as

$$
G=\frac{H(a)-H(a+\Delta a)}{\Delta a},
$$


where $H$ is given in Eq. (3), $\Delta a$ is the change in crack area $a$ (crack length in two dimensions) and the same electromechanical load is considered to compute $H(a)$ and $H(a+\Delta a)$. The four-point bending specimen in Fig. 3 with the material properties of PZT-PIC 151, poled along the $x_{2}$-axis, is considered (see Appendix B for the material parameters). We assume the free space as the crack-gap filling medium with $\varepsilon_{0}=8.854 \times 10^{-12} \mathrm{~F} / \mathrm{m}$. The energy-release rate is obtained as a function of the applied electric field considering the different crack-face conditions and two cracked test specimens with $a=0.8 \mathrm{~mm}$ and $0.85 \mathrm{~mm}$, respectively ( $\Delta a=0.05 \mathrm{~mm}$ ). The value of $a$ is the length of the region with $v=0$ (i.e. the crack length). The pre-cracks are introduced in the model using the profile in Eq. (2). The model is discretized with two different meshes, termed coarse and fine, with the smallest element size of $h=10^{-2} \mathrm{~mm}$ and $h=10^{-4} \mathrm{~mm}$ in the vicinity of the pre-crack, respectively. An adaptive mesh refinement algorithm is employed to generate the meshes. A typical mesh for $a=0.8 \mathrm{~mm}$ is presented in Fig. 4. The regularization parameter $\kappa$ is selected as four times the smallest element size and the residual stiffness is set to $\eta=10^{-6}$. A load $P=2000 \mathrm{~N}$ is considered and different electrical loads are applied in the $x_{2}$ direction. The mechanical displacements and electric potentials are obtained for the two cracked bodies using the weak form in Eq. (9) considering the stresses and electric displacements given for the different crack-face conditions in Table E2. The value of the total electromechanical enthalpy for each case is calculated from Eq. (3) using the corresponding enthalpy density and then the energy release rates are obtained from Eq. (C.1).

Fig. C1 presents the energy-release rates for the different crack-face conditions as a function of the applied electric field for the two mesh sizes. For the permeable crack, contrary to one's intuition, the energy release rate computed for the coarse mesh decreases as the magnitude of the applied electric field increases. This is due to the discontinuity in the electric displacement introduced through the coupling term $\mathbf{e}: \boldsymbol{\varepsilon}$ as pointed out in Section 4.1 .3 , cf. Eq. (15). This situation is readily corrected by refining the mesh and reducing $\kappa$ simultaneously. Indeed, for the resolved (fine) mesh it is interesting to observe that the energy release rate is insensitive to the magnitude of the applied electric field, in agreement with the results of the sharp-crack model obtained by Li et al. (2008). Thus, this result provides numerical evidence that, if the regularization parameter $\kappa$ tends to zero, the effect of the jump in the electric displacement disappears and the permeable conditions are satisfied with high accuracy. In contrast, the impermeable conditions lead to a significant decrease of the energy release rate with increasing applied electric field, resulting in a strong retarding effect on the crack propagation for high applied electric fields. The behavior of the EC model is similar to that of the impermeable model, with two main differences: (1) the retarding effect on the crack propagation is less significant, and (2) the behavior is asymmetric under positive (along the poling direction) and negative (opposite to the poling direction) applied electric fields, the retarding effect being weaker under negative applied electric fields. The converged results of the phase-field models corresponding to the different crack-face conditions for the fine mesh are in qualitative agreement with the results of the corresponding sharp-crack model obtained by Li et al. (2008). A quantitative comparison has not been pursued since the energy release rate was computed with an excessively large crack length increment $(\Delta a=0.5 \mathrm{~mm})$ in Li et al. (2008). It is worth mentioning that for large applied electric fields, the energy release rate for impermeable and EC cracks becomes negative, showing that there is no energetic incentive for the crack to propagate. We have discussed on a related phenomenon in the phase field in Section 4.4.

We next compare the profiles of stresses and electric displacements across the diffuse cracks for different values of the regularization parameter $\kappa$ and the mesh size $h$ with those obtained for the sharp-crack model. The goal is twofold: (1) to show that the phase-field solutions satisfy the corresponding crack-face boundary conditions, and (2) to show the convergence of the phase-field solutions to the sharp-crack solutions. Fig. C2 presents the results for the traction-free,

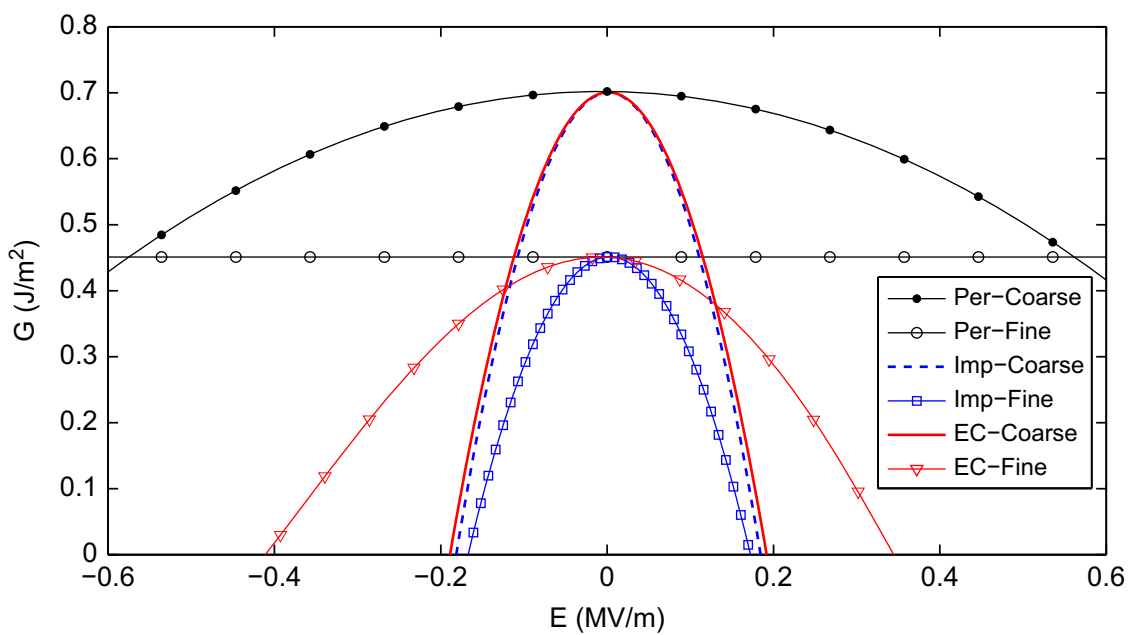

Fig. C1. Energy release rates in the piezoelectric material for the different crack face conditions: Permeable (Per), Impermeable (Imp) and air-filled Energetically Consistent (EC), as a function of applied electric field. A positive applied electric field is oriented in the poling direction. A mechanical load $P=2000 \mathrm{~N}$ is applied. The increment of crack length is $\Delta a=0.05$. The results are presented for a coarse mesh $\left(\kappa=4 \times 10^{-2} \mathrm{~mm}, h=10^{-2} \mathrm{~mm}\right)$ and a fine mesh $\left(\kappa=4 \times 10^{-4} \mathrm{~mm}, h=10^{-4} \mathrm{~mm}\right)$ 
a

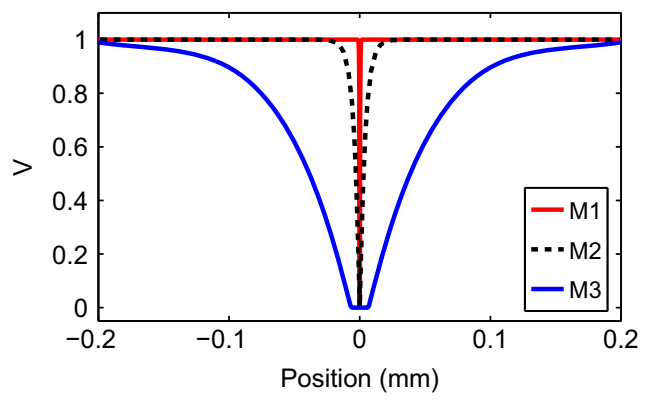

C

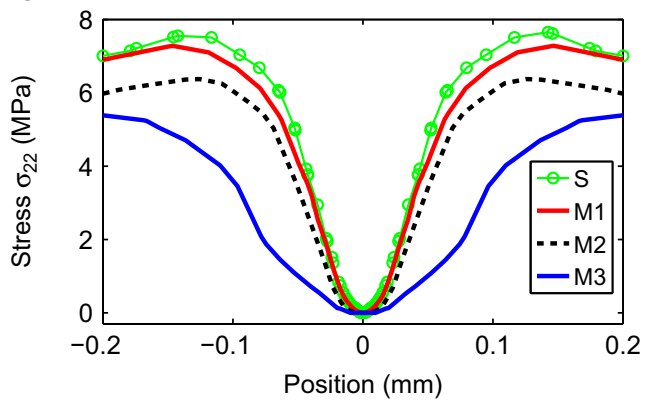

e

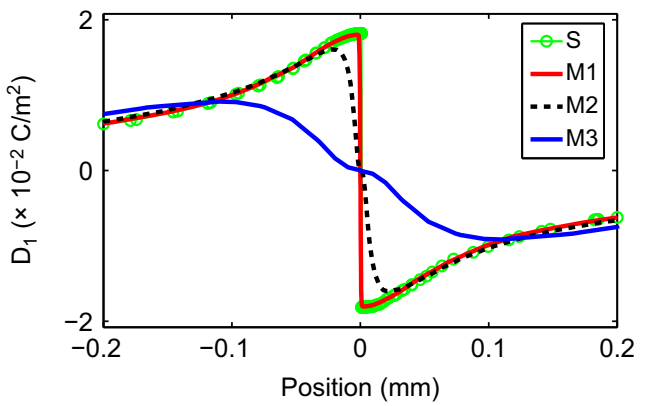

b

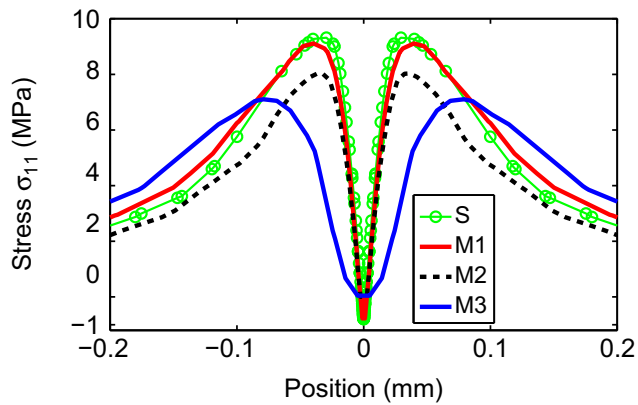

d

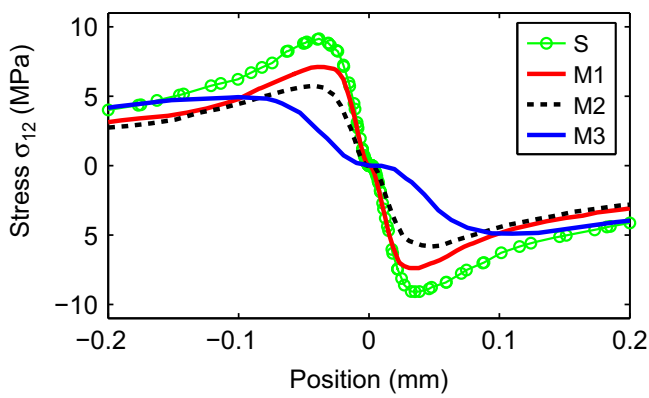

f

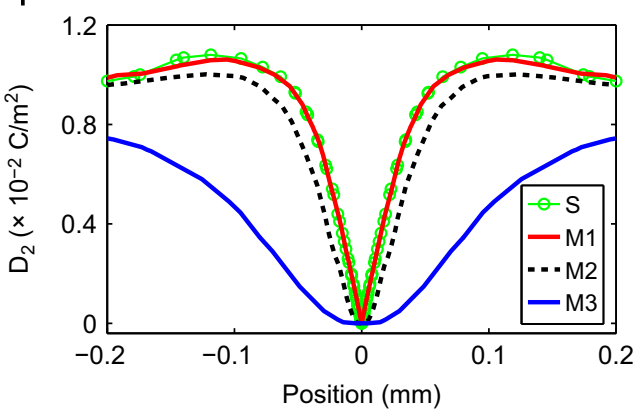

Fig. C2. In the piezoelectric material, (a) Field $v$ along a cross-section of the four-point bending sample in the $x_{2}$ direction (normal to the crack) behind the crack tip, (b)-(f) Stresses and electric displacements along the same section. The results are obtained for a traction-free, electrically impermeable precrack of length $a=1 \mathrm{~mm}$, an applied mechanical load $P=2000 \mathrm{~N}$ and no applied electric field. Different values of the regularization parameter $\kappa$ and mesh sizes $h$ are chosen near the smeared crack for each simulation: M1 $\left(\kappa=4 \times 10^{-2}, h=10^{-2}\right), \mathrm{M} 2(\kappa=0.4, h=0.1)$ and M3 ( $\left.\kappa=4, h=1\right)$. A simulation is also done for the sharp-crack model (marked with S) with mesh size $h=10^{-2}$ near the crack faces. Both $\kappa$ and $h$ are normalized by the value of $10^{-2}$ mm.

impermeable crack model along a cross section of the four-point bending sample in the $x_{2}$ direction (normal to the crack) behind the crack tip. The field $v$ is also plotted along the same section in Fig. C2(a) to indicate the cross-section of the diffuse cracks. A stationary pre-crack of length $a=1 \mathrm{~mm}$ is considered, and a point load $P=2000 \mathrm{~N}$ and no electric field $(\Delta V=0)$ are applied. For comparison purposes, the same problem is solved with the corresponding sharp-crack model by geometrically introducing a pre-crack of the same length in the computational model. It is obvious in Fig. C2 that by decreasing the value of the regularization parameter and the mesh size in a concerted manner, the transition of the field $v$ between the intact $(v=1)$ and fractured $(v=0)$ zones becomes sharper and the stresses and electric displacements recover the solutions of the sharp-crack model near the edges of the smeared crack. The $\sigma_{22}, \sigma_{12}$, and $D_{2}$ components in Fig. C2 vanish in the fractured zone $(v=0)$ and at the edges of the diffuse cracks to fulfill the traction-free and charge-free conditions of an impermeable crack, respectively. The other components $\sigma_{11}$ and $D_{1}$, not affected by these conditions, exhibit non-zero values at the edges of the smeared cracks. Similar convergence results for the traction-free, electrically permeable crack model are omitted here for the sake of brevity.

For the EC phase-field model, a direct comparison of the stresses and electric displacements solutions of the phase-field and the sharp-crack models involves solving a complex non-linear problem for the sharp-crack model. To avoid this computation, which is not the purpose of the present paper, we verify that the proposed electrical enthalpy of the fracture zone, $H_{c}$ in Eq. (24), converges to the enthalpy of the crack gap in Eq. (20) in the corresponding sharp-crack model by evaluating these crack gap enthalpies on generic states, which are not equilibrium states of the EC model. Here, we chose as equivalent states for this evaluation two equilibrium states of the impermeable phase-field and sharp-crack models. We choose the impermeable 


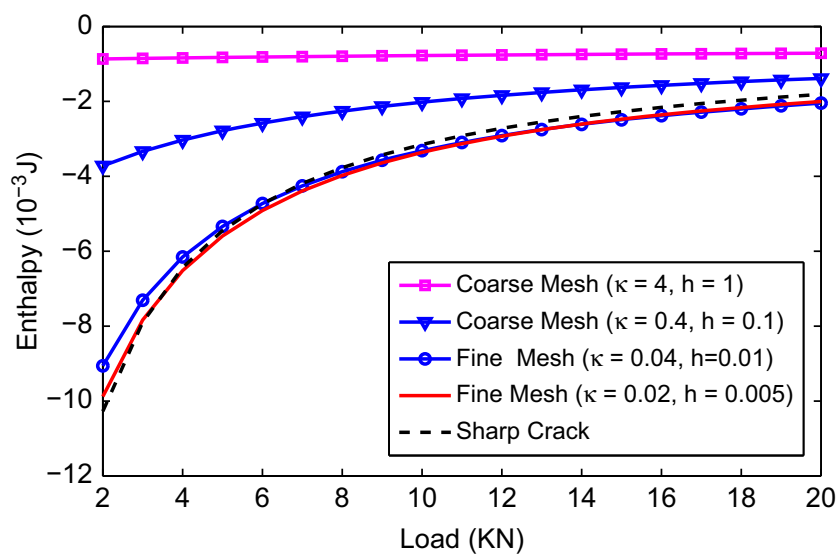

Fig. C3. Total electrical enthalpy of the diffuse crack in the piezoelectric material as a function of the mechanical load. Simulations are performed for different values of the regularization parameter $\kappa$ and mesh sizes $h$. A simulation is also done for the sharp-crack model with mesh size $h=5 \times 10^{-3}$ near the crack faces. The electrical load is fixed to a constant value of $E=0.7 \mathrm{MV} / \mathrm{m}$ for all the simulations. Both $\kappa$ and $h$ are normalized by the value of $10^{-2}$ mm.

crack model, since impermeable conditions induce a high electric field within the crack gap, consequently making the electrical enthalpy prominent in this zone. Simulations are performed using the four-point bending specimen in Fig. 3 with a pre-crack of length $a=1 \mathrm{~mm}$. The applied electric field is fixed to the constant value of $E=0.7 \mathrm{MV} / \mathrm{m}$ and the results are obtained for different applied point loads $P$. Fig. C3 presents the electrical enthalpy of the fracture zone for the diffuse crack as computed from $H_{c}$ in Eq. (24) for four different values of the regularization parameter $\kappa$ and the mesh size $h$, along with the enthalpy of the crack gap in the sharp-crack model as computed from Eq. (20). It is obvious in Fig. C3 that by decreasing the value of the regularization parameter $\kappa$ and mesh size $h$ in a concerted manner, the electrical enthalpy in the fracture zone converges to that of the sharp crack. The small difference between the results of the finest mesh and the sharp crack for large values of applied load is due to the components of the electric field parallel to the crack faces neglected in the sharp-crack model. Note that this result holds for the EC phase-field model of fracture in both piezoelectrics and ferroelectrics.

\section{C.2. Phase-field models for fracture in ferroelectrics}

For the phase-field models of fracture in ferroelectrics, we have conducted analogous verification tests. We have obtained similar conclusions from the comparison of the profiles of stresses and electric displacements across the diffuse crack with those computed for the sharp crack, for the case of the permeable and impermeable crack models and both for free-polarization and zero-polarization conditions. This is not surprising since the phase-field treatment of the crack face conditions is fundamentally the same for piezo- and ferroelectrics. Some of these results for impermeable, freepolarization crack conditions are presented in our recent work (Abdollahi and Arias, 2011a).

For the sake of brevity, we just present here a comparison of the polarization across stationary cracks obtained with the phase-field models for different values of the regularization parameter $\kappa$ and the mesh size $h$, and the equivalent sharp-crack models. A rectangular domain is considered with the loading, the boundary conditions and the pre-crack presented in Fig. 7 and with the material parameters of single crystals of barium titanate $\left(\mathrm{BaTiO}_{3}\right)$, see Appendix A. The domain is discretized such that smaller elements are located near the pre-crack. The upper and bottom surfaces are subjected to a uniform normalized tensile stress $\sigma_{22}^{\prime}=0.7$. The electric potential $\phi$ is set to zero in the upper and lower boundaries ( $\left.E=0\right)$. Traction free and open circuited $(\boldsymbol{D} \cdot \boldsymbol{n}=0)$ conditions are considered on the lateral faces. We also consider free-polarization conditions in all the boundary of the rectangular domain. In these simulations, the field $v$ is kept fixed, and Algorithm 1 is executed without solving Eq. (36). In this way, the initial polarization $\mathbf{p}_{0}$ is relaxed around the stationary pre-crack, which requires $5 \times 10^{3}$ time steps of normalized length $\Delta t^{\prime}=10^{-2}$. The regularization parameter for fracture $\kappa$ is set to four times of the smallest element near the pre-crack, the residual stiffness to $\eta=10^{-6}$, and the inverse mobility of the polarization evolution to $\mu_{p}=5 \times 10^{-2}$. We consider the free-polarization and zero-polarization crack models along with traction-free and electrically impermeable crack-face conditions. The electromechanical enthalpy density of these two models are given in Table E3. Fig. C4 presents the relaxed polarization along a cross-section of the ferroelectric sample behind the crack tip. For the free-polarization model, Fig. C4(a) and (b) shows that the components of the polarization approach with zero slope the edges of the smeared crack, in agreement with the results of the sharp-crack model. On the other hand, in the zeropolarization model, both polarization components tend to zero when approaching the crack edges, see Fig. C4(c) and (d). Furthermore, the polarization profile converges to that of the sharp-crack model as the regularization parameter $\kappa$ and the mesh size $h$ decrease. The vertical components of the polarization for both models vanish near the edges of the crack since the impermeable crack-face conditions force the polarization to be parallel to the crack. 
a

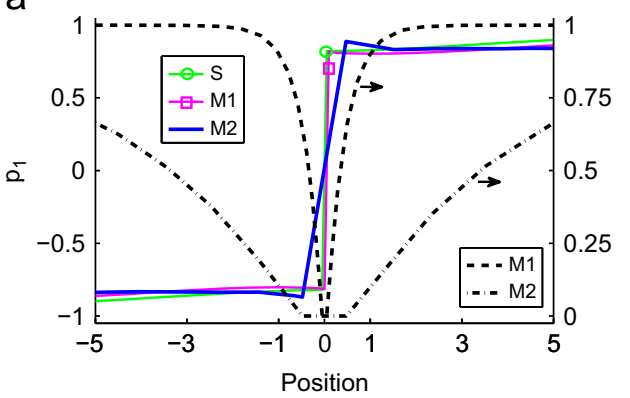

C

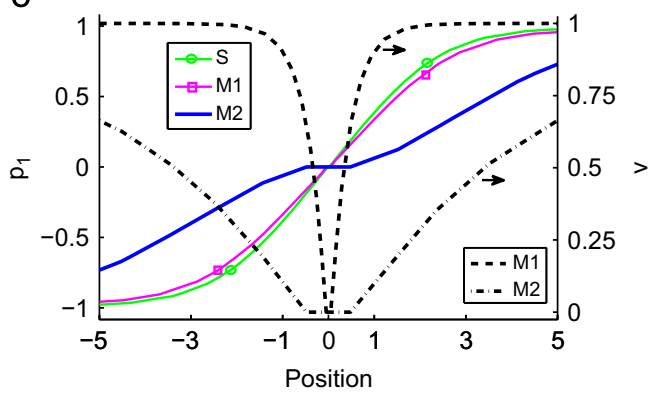

b

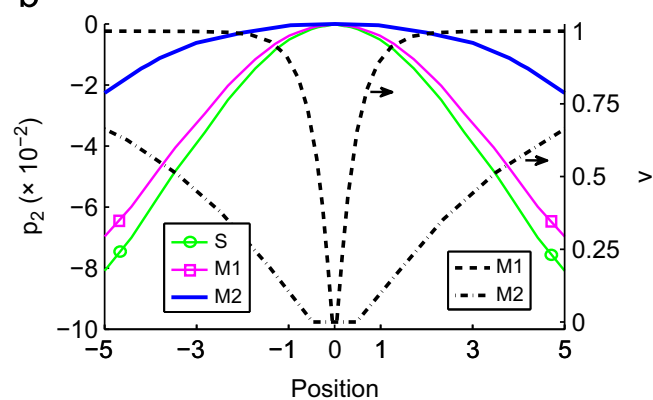

d

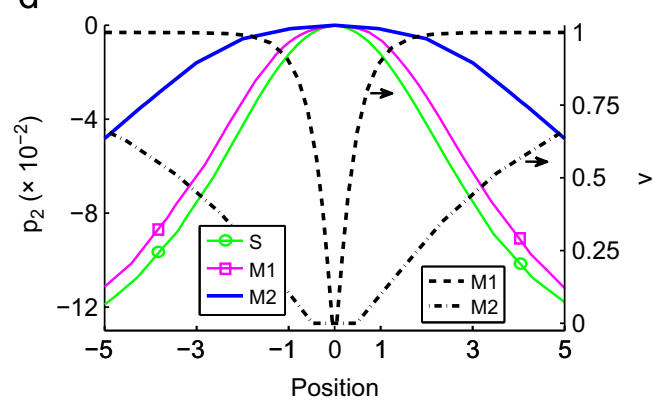

Fig. C4. Relaxed polarization along a cross-section of the ferroelectric sample perpendicular to the pre-crack and behind the crack tip: (a) horizontal polarization $\mathbf{p}_{1}$, (b) vertical polarization $\mathbf{p}_{2}$ in the free-polarization crack model and (c) $\mathbf{p}_{1}$, (d) $\mathbf{p}_{2}$ in the zero-polarization crack model. Results are presented for different values of the regularization parameter $\kappa$ and of the mesh size $h$ near the smeared crack: M1 $(\kappa=0.4, h=0.1)$, M2 $(\kappa=4, h=1)$ A simulation is also performed for the sharp-crack model (labeled with S) with mesh size $h=0.1$ near the crack faces. The field $v$ is plotted along the same section for each mesh to indicate the cross-section of the diffuse cracks.

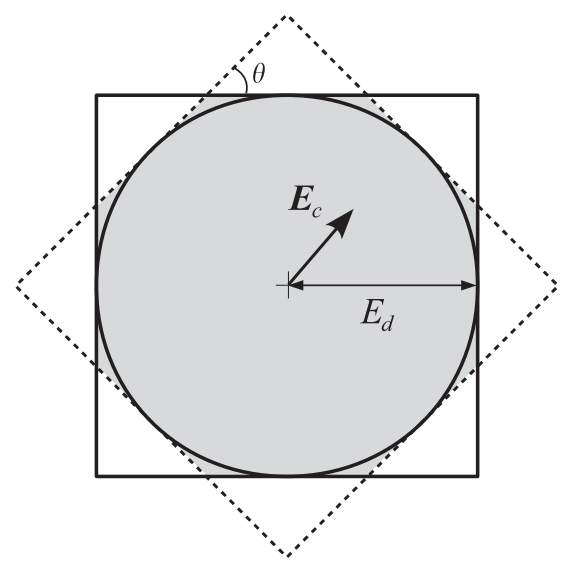

Fig. D1. A schematic of the electrical breakdown constraint and its linearization. The circle with the radius of the dielectric strength $E_{d}$ indicates the nonlinear constraint for the electric field $\boldsymbol{E}_{c}$. The highlighted gray octagon presents a linear approximation of this constraint. The origin of the coordinate system and $\boldsymbol{E}_{c}$ is the center of the circle.

\section{Appendix D. Constraint of electrical breakdown}

The constraint of electrical breakdown in Eq. (32) is a nonlinear inequality constraint for the optimization of the energy functional. To avoid the complexity in solving this nonlinear optimization problem, we linearize the inequality constraint as follows. The nonlinear constraint forces the electric field $\boldsymbol{E}_{c}$ to lie in a circle with the radius of the dielectric strength $E_{d}$ presented in Fig. D1. A convenient linear approximation of this circle can be defined by the intersection of squares rotated a constant angle $\theta$. Fig. D1 presents a schematic of this linearization by two squares with the rotation angle of $\theta=45^{\circ}$. The first and second squares with line- and dashed-borders impose linear inequality constraints for the electric field $\boldsymbol{E}_{c}$, respectively as

$$
\begin{aligned}
& -E_{d} \leq A_{i} \leq E_{d}, \\
& -E_{d} \leq B_{i} \leq E_{d},
\end{aligned}
$$


Table E1

Crack-face boundary conditions in electromechanical materials.

\begin{tabular}{|c|c|c|c|}
\hline \multicolumn{3}{|c|}{ Crack-face boundary conditions } & \multirow{2}{*}{$\begin{array}{l}\text { Mathematical description } \\
\boldsymbol{\sigma}^{+} \cdot \boldsymbol{n}=\boldsymbol{\sigma}^{-} \cdot \boldsymbol{n}=\mathbf{0} \\
\phi^{+}=\phi^{-}, \boldsymbol{D}^{+} \cdot \boldsymbol{n}=\boldsymbol{D}^{-} \cdot \boldsymbol{n} \\
\boldsymbol{D}^{+} \cdot \boldsymbol{n}=\boldsymbol{D}^{-} \cdot \boldsymbol{n}=0\end{array}$} \\
\hline Uncoupled & $\begin{array}{l}\text { Mechanical } \\
\text { Electrical }\end{array}$ & $\begin{array}{l}\text { Traction-free } \\
\text { Permeable } \\
\text { Impermeable }\end{array}$ & \\
\hline Coupled & Electro-mechanical & Energetically consistent (EC) & $\begin{array}{l}\boldsymbol{D}^{+} \cdot \boldsymbol{n}=\boldsymbol{D}^{-} \cdot \boldsymbol{n}=\varepsilon_{0} E_{c} \\
\sigma_{c}=\varepsilon_{0} E_{c}^{2} / 2 \\
\mathcal{H}_{c}=-\varepsilon_{0} E_{c}^{2} / 2\end{array}$ \\
\hline Polarization & $\begin{array}{l}\text { Free-polarization } \\
\text { Zero-polarization }\end{array}$ & & $\begin{array}{l}\nabla \mathbf{p}^{+} \cdot \boldsymbol{n}=\nabla \mathbf{p}^{-} \cdot \boldsymbol{n}=\mathbf{0} \\
\mathbf{p}^{+}=\mathbf{p}^{-}=\mathbf{0}\end{array}$ \\
\hline \multicolumn{4}{|c|}{$\begin{array}{l}\text { p: Polarization, } \boldsymbol{\sigma} \text { : Stress, } \boldsymbol{D} \text { : Electric displacement, } \phi \text { : Electric potential } \\
\boldsymbol{n} \text { : Unit normal to the top }(+) \text { and bottom }(-) \text { crack faces } \\
E_{c} \text { and } \sigma_{c} \text { : Electric field and stress induced in the crack gap } \\
\mathcal{H}_{c} \text { : Electrical enthalpy density of the crack gap }\end{array}$} \\
\hline
\end{tabular}

\section{Table E2}

Electromechanical enthalpy density, stresses and electric displacements for piezoelectric materials with different electromechanical crack conditions.

Per

$\mathcal{H}=\left(v^{2}+\eta_{\kappa}\right)\left(\frac{1}{2} \boldsymbol{\varepsilon}: \mathbb{C}: \boldsymbol{\varepsilon}-\boldsymbol{\varepsilon}: \boldsymbol{e}^{T} \cdot \boldsymbol{E}\right)-\frac{1}{2} \boldsymbol{E} \cdot \boldsymbol{K} \boldsymbol{E}$,

$\boldsymbol{\sigma}=\left(v^{2}+\eta_{\kappa}\right)\left(\mathbb{C}: \boldsymbol{\varepsilon}-\boldsymbol{e}^{T} \cdot \boldsymbol{E}\right), \quad \boldsymbol{D}=\left(v^{2}+\eta_{\kappa}\right) \boldsymbol{e}: \boldsymbol{\varepsilon}+\boldsymbol{K} \boldsymbol{E}$

$\operatorname{Imp}$

$$
\begin{aligned}
& \mathcal{H}^{*}=\left(v^{2}+\eta_{k}\right)\left(\frac{1}{2} \boldsymbol{\varepsilon}: \mathbb{C}: \boldsymbol{\varepsilon}-\boldsymbol{\varepsilon}: \boldsymbol{e}^{T} \cdot \boldsymbol{E}-\frac{1}{2} \boldsymbol{E} \cdot \boldsymbol{K} \boldsymbol{E}\right), \\
& \boldsymbol{\sigma}^{*}=\left(v^{2}+\eta_{\kappa}\right)\left(\mathbb{C}: \boldsymbol{\varepsilon}-\boldsymbol{e}^{T} \cdot \boldsymbol{E}\right), \quad \boldsymbol{D}^{*}=\left(v^{2}+\eta_{\kappa}\right)(\boldsymbol{e}: \boldsymbol{\varepsilon}+\boldsymbol{K} \boldsymbol{E})
\end{aligned}
$$

EC

$$
\begin{aligned}
& \mathcal{H}=\mathcal{H}^{*}-\frac{\varepsilon_{0}}{2}\left(1-v^{2}\right)\left|\boldsymbol{F}^{-T} \boldsymbol{E}\right|^{2} J, \\
& \boldsymbol{\sigma}=\boldsymbol{\sigma}^{*}+\frac{J}{2}\left(1-v^{2}\right) \boldsymbol{\sigma}_{c}, \quad \boldsymbol{D}=\boldsymbol{D}^{*}+J\left(1-v^{2}\right) \boldsymbol{F}^{-1} \boldsymbol{D}_{c}
\end{aligned}
$$

Per: Permeable (traction-free), Imp: Impermeable (traction-free), EC: Energetically consistent.

where $A_{i}$ and $B_{i}(i=1,2)$ are the components of the vector $\boldsymbol{E}_{c}$ and $\boldsymbol{R} \boldsymbol{E}_{c}$, respectively, $\boldsymbol{R}$ being the rotation matrix associated with the rotation angle $\theta$. Combining Eqs. (D.1) and (D.2) leads to a linear constraint as

$$
-E_{d} \leq C_{i} \leq E_{d}
$$

where $C_{i}(i=1, \ldots, 4)$ are the components of the vector $[\boldsymbol{I} \boldsymbol{R}]^{T} \boldsymbol{E}_{c}$, I being the two dimensional identity matrix. In fact, the inequality constraint in Eq. (D.3) spans the intersection of the two squares which is an octagon highlighted in gray in Fig. D1 and it approximates the circular area imposed by the constraint of electrical breakdown in Eq. (32). It is obvious that this approximation becomes more accurate with increasing number of squares, whose intersection creates a regular high order polygon. However, this results in an increase in the size of the vector $\boldsymbol{C}$ which in turn magnifies the computational cost of the optimization problem. To apply the constraint in Eq. (D.3), we use quadratic programming (Gertz and Wright, 2001, 2003). Since the constraint of electrical breakdown is an inequality constraint for the electric field, the Lagrange multipliers can be physically interpreted as additional charges in the fracture zone penalizing the excess of the electric field above the dielectric strength $E_{d}$. Finally, we note that the product of the vector $\boldsymbol{C}$ by the jump set function $\left(1-v^{2}\right)$ restricts the constraint of electrical breakdown to the fracture zone. 
Table E3

Electromechanical enthalpy density, stresses and electric displacements for ferroelectric materials with different electromechanical crack conditions.

Free-polarization

Per

$$
\begin{aligned}
& \mathcal{H}=\left(v^{2}+\eta_{\kappa}\right)\left(\frac{1}{2} \boldsymbol{\varepsilon}: \mathbb{C}: \boldsymbol{\varepsilon}-\boldsymbol{\varepsilon}: \mathbb{C}: \boldsymbol{\varepsilon}_{s}+U\right)+\chi-\boldsymbol{E} \cdot \mathbf{p}-\frac{\varepsilon_{0}}{2}|\boldsymbol{E}|^{2}, \\
& \boldsymbol{\sigma}=\left(v^{2}+\eta_{\kappa}\right)\left[\mathbb{C}:\left(\boldsymbol{\varepsilon}-\boldsymbol{\varepsilon}_{s}\right)\right], \quad \boldsymbol{D}=\mathbf{p}+\varepsilon_{0} \boldsymbol{E}
\end{aligned}
$$

Imp

$$
\begin{aligned}
& \mathcal{H}^{*}=\left(v^{2}+\eta_{\kappa}\right)\left(\frac{1}{2} \boldsymbol{\varepsilon}: \mathbb{C}: \boldsymbol{\varepsilon}-\boldsymbol{\varepsilon}: \mathbb{C}: \boldsymbol{\varepsilon}_{s}+U-\boldsymbol{E} \cdot \mathbf{p}-\frac{\varepsilon_{0}}{2}|\boldsymbol{E}|^{2}\right)+\chi \\
& \boldsymbol{\sigma}^{*}=\left(v^{2}+\eta_{\kappa}\right)\left[\mathbb{C}:\left(\boldsymbol{\varepsilon}-\boldsymbol{\varepsilon}_{s}\right)\right], \quad \boldsymbol{D}^{*}=\left(v^{2}+\eta_{\kappa}\right)\left(\mathbf{p}+\varepsilon_{0} \boldsymbol{E}\right)
\end{aligned}
$$

EC

$$
\begin{aligned}
& \mathcal{H}=\mathcal{H}^{*}-\frac{\varepsilon_{0}}{2}\left(1-v^{2}\right)\left|\boldsymbol{F}^{-T} \boldsymbol{E}\right|^{2} J, \\
& \boldsymbol{\sigma}=\boldsymbol{\sigma}^{*}+\frac{J}{2}\left(1-v^{2}\right) \boldsymbol{\sigma}_{c}, \quad \boldsymbol{D}=\boldsymbol{D}^{*}+J\left(1-v^{2}\right) \boldsymbol{F}^{-1} \boldsymbol{D}_{c}
\end{aligned}
$$

Zero-polarization

Per

$$
\begin{aligned}
\mathcal{H} & =\left(v^{2}+\eta_{\kappa}\right)\left(\frac{1}{2} \boldsymbol{\varepsilon}: \mathbb{C}: \boldsymbol{\varepsilon}-\boldsymbol{\varepsilon}: \mathbb{C}: \boldsymbol{\varepsilon}_{s}+\chi-\boldsymbol{E} \cdot \mathbf{p}\right)+U-\frac{\varepsilon_{0}}{2}|\boldsymbol{E}|^{2} \\
& -\left(1-v^{2}\right) \frac{\hat{\varepsilon}-\varepsilon_{0}}{2}|\boldsymbol{E}|^{2}, \quad \boldsymbol{\sigma}=\left(v^{2}+\eta_{\kappa}\right)\left[\mathbb{C}:\left(\boldsymbol{\varepsilon}-\boldsymbol{\varepsilon}_{s}\right)\right], \\
\boldsymbol{D} & =\left(v^{2}+\eta_{\kappa}\right) \mathbf{p}+\varepsilon_{0} \boldsymbol{E}+\left(1-v^{2}\right)\left(\hat{\varepsilon}-\varepsilon_{0}\right) \boldsymbol{E}
\end{aligned}
$$

Imp

$$
\begin{aligned}
& \mathcal{H}^{*}=\left(v^{2}+\eta_{\kappa}\right)\left(\frac{1}{2} \boldsymbol{\varepsilon}: \mathbb{C}: \boldsymbol{\varepsilon}-\boldsymbol{\varepsilon}: \mathbb{C}: \boldsymbol{\varepsilon}_{s}+\chi-\boldsymbol{E} \cdot \mathbf{p}-\frac{\varepsilon_{0}}{2}|\boldsymbol{E}|^{2}\right)+U, \\
& \boldsymbol{\sigma}^{*}=\left(v^{2}+\eta_{\kappa}\right)\left[\mathbb{C}:\left(\boldsymbol{\varepsilon}-\boldsymbol{\varepsilon}_{s}\right)\right], \quad \boldsymbol{D}^{*}=\left(v^{2}+\eta_{\kappa}\right)\left(\mathbf{p}+\varepsilon_{0} \boldsymbol{E}\right)
\end{aligned}
$$

EC

$$
\begin{aligned}
& \mathcal{H}=\mathcal{H}^{*}-\frac{\varepsilon_{0}}{2}\left(1-v^{2}\right)\left|\boldsymbol{F}^{-T} \boldsymbol{E}\right|^{2} J, \\
& \boldsymbol{\sigma}=\boldsymbol{\sigma}^{*}+\frac{J}{2}\left(1-v^{2}\right) \boldsymbol{\sigma}_{c}, \quad \boldsymbol{D}=\boldsymbol{D}^{*}+J\left(1-v^{2}\right) \boldsymbol{F}^{-1} \boldsymbol{D}_{c}
\end{aligned}
$$

Per: Permeable (traction-free), Imp: Impermeable (traction-free), EC: Energetically consistent.

\section{Appendix E. Electromechanical enthalpy density, stresses and electric displacements for piezoelectric and ferroelectric materials with different electromechanical crack conditions}

We summarize in Table E1, the crack-face boundary conditions in electromechanical materials discussed in Sections 1 and 4. Based on these conditions, different electromechanical enthalpy density $\mathcal{H}$, stresses $\boldsymbol{\sigma}$, and electric displacements $\boldsymbol{D}$ are obtained and summarized for piezoelectric and ferroelectric materials in Tables E2 and E3, respectively. All the parameters are defined in Sections 2, 3, and 4. $\boldsymbol{D}_{c}$ and $\boldsymbol{\sigma}_{c}$ are given in Eqs. (30) and (31), respectively. $\hat{\varepsilon}$ is the dielectric constant of the ferroelectric material.

\section{References}

Abdollahi, A., Arias, I., 2011a. Phase-field modeling of the coupled microstructure and fracture evolution in ferroelectric single crystals. Acta Mater. 59, 4733-4746.

Abdollahi, A., Arias, I., 2011b. Phase-field simulation of anisotropic crack propagation in ferroelectric single crystals: effect of microstructure on the fracture process. Model. Simul. Mater. Sci. Eng. 19, 074010.

Abdollahi, A., Arias, I., 2012. Numerical simulation of intergranular and transgranular crack propagation in ferroelectric polycrystals. Int. J. Fract. 174, 3-15.

Amor, H., Marigo, J.J., Maurini, C., 2009. Regularized formulation of the variational brittle fracture with unilateral contact: numerical experiments. J. Mech. Phys. Solids 57, 1209-1229.

Anderson, T.L., 1991. Fracture Mechanics Fundamental and Applications. CRC Press.

Arias, I., Serebrinsky, S., Ortiz, M., 2006. A phenomenological cohesive model of ferroelectric fatigue. Acta Mater. 54, 975-984. 
Beom, H.G., Atlurib, S.N., 2003. Effect of electric fields on fracture behavior of ferroelectric ceramics. J. Mech. Phys. Solids 51, 1107-1125.

Bourdin, B., 2007. Numerical implementation of the variational formulation for quasi-static brittle fracture. Interfaces Free Bound. 9, 411-430.

Bourdin, B., Francfort, G.A., Marigo, J.J., 2000. Numerical experiments in revisited brittle fracture. J. Mech. Phys. Solids 48, 797-826.

Bourdin, B., Francfort, G.A., Marigo, J.J., 2008. The variational approach to fracture. J. Elast. 91, 5-148.

Camacho, G.T., Ortiz, M., 1996. Computational modelling of impact damage in brittle materials. Int. J. Numer. Methods Eng. 33, $2899-2938$.

Dayal, K., Bhattacharya, K., 2006. Kinetics of phase transformations in the peridynamic formulation of continuum mechanics. J. Mech. Phys. Solids 54, $1811-1842$.

Deeg, W.F.J., 1980. The Analysis of Dislocation, Crack, and Inclusion Problems in Piezoelectric Solids. Ph.D. Thesis. Stanford University.

DeSimone, A., James, R.D., 2002. A constrained theory of magnetoelasticity. J. Mech. Phys. Solids 50, 283-320.

DeSimone, A., Kohn, R.V., Müller, S., Otto, F., Schäfer, R., 2001. Two-dimensional modeling of soft ferromagnetic films. Proc. R. Soc. London A 457, 2983-2991.

Devonshire, A.F., 1949. Theory of barium titanate 1. Philos. Mag. 40, 1040-1063.

Devonshire, A.F., 1951. Theory of barium titanate 2. Philos. Mag. 42, 1065-1079.

Engert, A.R., Neumeister, P., Mecklenburg, M., Jelitto, H., Balke, H., Schneider, G.A., 2011. Influence of small cyclic and dc electrical loads on the fracture toughness of ferroelectric ceramics. J. Eur. Ceram. Soc. 31, 531-540.

Fang, D., Jiang, Y., Li, S., Sun, C.T., 2007. Interactions between domain switching and crack propagation in poled BaTiO ${ }_{3}$ single crystal under mechanical loading. Acta Mater. 55, 5758-5767.

Floquet, N., Valot, C.M., Mesnier, M.T., Niepce, J.C., Normand, L., Thorel, A., Kilaas, R., 1997. Ferroelectric domain walls in BaTiO ${ }_{3}$ : fingerprints in XRPD diagrams and quantitative HRTEM image analysis. J. Phys. III 7, 1105-1128.

Francfort, G.A., Marigo, J.J., 1998. Revisiting brittle fracture as an energy minimization problem. J. Mech. Phys. Solids 46, 1319-1342.

Fu, R., Zhang, T.Y., 2000. Effects of an electric field on the fracture toughness of poled lead zirconate titanate ceramics. J. Am. Ceram. Soc. 83, $1215-1218$.

Gao, H.J., Zhang, T.Y., Tong, P., 1997. Local and global energy release rates for an electrically yielded crack in a piezoelectric ceramic. J. Mech. Phys. Solids 45, 491-510.

Gertz, E.M., Wright, S.J., 2001. OOQP User Guide. Argonne National Laboratory.

Gertz, E.M. Wright, S.J., 2003. Object-oriented software for quadratic programming. ACM Trans. Math. Software 29, 58-81.

Griffith, A.A., 1921. The phenomena of rupture and flow in solids. Philos. Trans. R. Soc. London Ser. A 221, 163-198.

Hackemann, S., Pfeiffer, W., 2003. Domain switching in process zones of PZT: characterization by microdiffraction and fracture mechanical methods. J. Eur. Ceram. Soc. 23, 141-151.

Hakim, V., Karma, A., 2009. Laws of crack motion and phase-field models of fracture. J. Mech. Phys. Solids 57, 342-368.

Hao, T.H., Shen, Z.Y., 1994. A new electric boundary-condition of electric fracture-mechanics and its applications. Eng. Fract. Mech. 47, 793-802.

Haug, A., McMeeking, R.M., 2006. Cracks with surface charge in poled ferroelectrics. Eur. J. Mech. A Solids 25, 24-41.

Heyer, V., Schneider, G.A., Balke, H., Drescher, J., Bahr, H.A., 1998. A fracture criterion for conducting cracks in homogeneously poled piezoelectric PZT-PIC 151 ceramics. Acta Mater. 46, 6615-6622.

Huber, J.E., 2005. Micromechanical modelling of ferroelectrics. Curr. Opin. Solid State Mater. Sci. 9, 100-106.

Hwang, S.C., Lynch, C.S., McMeeking, R.M., 1995. Ferroelectric/ferroelastic interactions and a polarization switching model. Acta Mater. 43, $2073-2084$.

Jiang, Y., Zhang, Y., Liu, B., Fang, D., 2009. Study on crack propagation in ferroelectric single crystal under electric loading. Acta Mater. 57, $1630-1638$.

Jones, L.J., Motahari, S.M., VarlioglU, M., Lienert, U., Bernier, J.V., Hoffman, M., Uestuendag, E., 2007. Crack tip process zone domain switching in a soft lead zirconate titanate ceramic. Acta Mater. 55, 5538-5548.

Kamlah, M., 2001. Ferroelectric and ferroelastic piezoceramics-modeling of electromechanical hysteresis phenomena. Contin. Mech. Thermodyn. 13, 219-268.

Kuhn, C., Müller, R., 2010. A continuum phase field model for fracture. Eng. Fract. Mech. 77, 3625-3634.

Kuna, M., 2010. Fracture mechanics of piezoelectric materials-where are we right now? Eng. Fract. Mech. 77, 309-326.

Landis, C.M., 2003. On the fracture toughness of ferroelastic materials. J. Mech. Phys. Solids 51, 1347-1369.

Landis, C.M., 2004a. Energetically consistent boundary conditions for electromechanical fracture. Int. J. Solids Struct. 41, 6291-6315.

Landis, C.M., 2004b. Non-linear constitutive modeling of ferroelectrics. Curr. Opin. Solid State Mater. Sci. 8, 59-69.

Li, J., Liu, D., 2004. On ferroelectric crystals with engineered domain configurations. J. Mech. Phys. Solids 52, 1719-1742.

Li, Q., Kuna, M., 2012. Evaluation of electromechanical fracture behavior by configurational forces in cracked ferroelectric polycrystals. Comput. Mater. Sci. $57,94-101$.

Li, W., Landis, C.M., 2011. Nucleation and growth of domains near crack tips in single crystal ferroelectrics. Eng. Fract. Mech. 78, $1505-1513$.

Li, W.Y., McMeeking, R.M., Landis, C.M., 2008. On the crack face boundary conditions in electromechanical fracture and an experimental protocol for determining energy release rates. Eur. J. Mech. A Solids 27, 285-301.

Li, Y.L., Cross, L.E., Chen, L.Q., 2005. A phenomenological thermodynamic potential for $\mathrm{BaTiO}_{3}$ single crystals. J. Appl. Phys. 98, 064101.

Linder, C., Rosato, D., Miehe, C., 2011. New finite elements with embedded strong discontinuities for the modeling of failure in electromechanical coupled solids. Comput. Methods Appl. Mech. Eng. 200, 141-161.

Linder, C., Miehe, C., 2012. Effect of electric displacement saturation on the hysteretic behavior of ferroelectric ceramics and the initiation and propagation of cracks in piezoelectric ceramics. J. Mech. Phys. Solids, 60, 882-903.

Loge, R.E., Suo, Z., 1996. Nonequilibrium thermodynamics of ferroelectric domain evolution. Acta Mater. 44, 3429-3438.

McMeeking, R.M., 1999. Crack tip energy release rate for a piezoelectric compact tension specimen. Eng. Fract. Mech. 64, 217-244.

McMeeking, R.M., 2004. The energy release rate for a Griffith crack in a piezoelectric material. Eng. Fract. Mech. 71, 1149-1163.

McMeeking, R.M., Landis, C.M., 2002. A phenomenological multi-axial constitutive law for switching in polycrystalline ferroelectric ceramics. Int. J. Eng. Sci. $40,1553-1577$.

Meschke, F., Raddatz, O., Kolleck, A., Schneider, G.A., 2000. R-curve behavior and crack-closure stresses in barium titanate and (Mg,Y)-PSZ ceramics. J. Am. Ceram. Soc. 83, 353-361.

Miehe, C., Welschinger, F., Hofacker, M., 2010. A phase field model of electromechanical fracture. J. Mech. Phys. Solids 58, $1716-1740$.

Moes, N., Dolbow, J., Belytschko, T., 1999. A finite element method for crack growth without remeshing. Int. J. Numer. Methods Eng. 46, 131-150.

Oliver, J., Huespe, A.E., Pulido, M.D.G., Chaves, E., 2002. From continuum mechanics to fracture mechanics: the strong discontinuity approach. Eng. Fract. Mech. 69, 113-136.

Parton, V.Z., 1976. Fracture mechanics of piezoelectric materials. Acta Astronaut. 3, 671-683.

Pojprapai, S., Jones, L.J., Vodenitcharova, T., Bernier, J.V., Hoffman, M., 2011. Investigation of the domain switching zone near a crack tip in pre-poled lead zirconate titanate ceramic via in situ X-ray diffraction. Scr. Mater. 64, 1-4.

Ricoeur, A., Kuna, M., 2003. Influence of electric fields on the fracture of ferroelectric ceramics. J. Eur. Ceram. Soc. 23, 1313-1328.

Schneider, G.A., 2007. Influence of electric field and mechanical stresses on the fracture of ferroelectrics. Annu. Rev. Mater. Res. 37, 491-538.

Schneider, G.A., Felten, F., McMeeking, R.M., 2003. The electrical potential difference across cracks in PZT measured by Kelvin Probe Microscopy and the implications for fracture. Acta Mater. 51, 2235-2241.

Schrade, D., Mueller, R., Xu, B.X., Gross, D., 2007. Domain evolution in ferroelectric materials: a continuum phase field model and finite element implementation. Comput. Methods Appl. Mech. Eng. 196, 4365-4374.

Sheng, J.S., Landis, C.M., 2007. Toughening due to domain switching in single crystal ferroelectric materials. Int. J. Fract. 143, 161-175.

Shindo, Y., Murakami, H., Horiguchi, K., Narita, F., 2002. Evaluation of electric fracture properties of piezoelectric ceramics using the finite element and single-edge precracked-beam methods. J. Am. Ceram. Soc. 85, 1243-1248. 
Shu, Y.C., Bhattacharya, K., 2001. Domain patterns and macroscopic behaviour of ferroelectric materials. Philos. Mag. B 81, $2021-2054$.

Silling, S.A., 2000. Reformulation of elasticity theory for discontinuities and long-range forces. J. Mech. Phys. Solids 48, $175-209$.

Song, Y.C., Soh, A.K., Ni, Y., 2007. Phase field simulation of crack tip domain switching in ferroelectrics. J. Phys. D Appl. Phys. 40, 1175-1182.

Stemmer, S., Streiffer, S.K., Ernst, F., Ruhle, M., 1995. Atomistic structure of 90-degrees domain-walls in ferroelectric PbTiO3 thin-films. Philos. Mag. 71, $713-724$

Su, Y., Landis, C.M., 2007. Continuum thermodynamics of ferroelectric domain evolution: theory, finite element implementation, and application to domain wall pinning. J. Mech. Phys. Solids 55, 280-305.

Tieresten, H.F., 1969. Linear Piezoelectric Plate Vibrations. Plenum Press.

Tipler, P.A., 1987. College Physics. Worth Publication.

Vendik, O.G., Zubko, S.P., 2000. Ferroelectric phase transition and maximum dielectric permittivity of displacement type ferroelectrics $\left(\mathrm{Ba}_{x} \mathrm{Sr}_{1-x} \mathrm{TiO}_{3}\right)$. J. Appl. Phys. 88, 5343-5350.

Völker, B., Marton, P., Elsässer, C., Kamlah, M., 2011. Multiscale modeling for ferroelectric materials: a transition from the atomic level to phase-field modeling. Contin. Mech. Thermodyn. 23, 435-451.

Wang, H.Y., Singh, R.N., 1997. Crack propagation in piezoelectric ceramics: effects of applied electric fields. J. Appl. Phys. 81, 7471-7479.

Wang, J., Kamlah, M., 2009. Three-dimensional finite element modeling of polarization switching in a ferroelectric single domain with an impermeable notch. Smart Mater. Struct. 18, 104008.

Wang, J., Kamlah, M., 2010. Effect of electrical boundary conditions on the polarization distribution around a crack embedded in a ferroelectric single domain. Eng. Fract. Mech. 77, 3658-3669.

Wang, J., Landis, C.M., 2006. Effects of in-plane electric fields on the toughening behavior of ferroelectric ceramics. J. Mech. Mater. Struct. 1, 1075-1095.

Wang, J., Zhang, T.Y., 2007. Phase field simulations of polarization switching-induced toughening in ferroelectric ceramics. Acta Mater. 55, 2465-2477.

Wang, Y.L., Tagantsev, A.K., Damjanovic, D., Setter, N., Yarmarkin, V.K., Sokolov, A.I., Lukyanchuk, I.A., 2007. Landau thermodynamic potential for BaTiO 3. J. Appl. Phys. 101, 104115.

Xiao, Y., Bhattacharya, K., 2008. A continuum theory of deformable, semiconducting ferroelectrics. Arch. Ration. Mech. Anal. 189, 59-95.

Xu, B.X., Schrade, D., Gross, D., Mueller, R., 2010a. Fracture simulation of ferroelectrics based on the phase field continuum and a damage variable. Int. J. Fract. 166, 163-172.

Xu, B.X., Schrade, D., Gross, D., Mueller, R., 2010b. Phase field simulation of domain structures in cracked ferroelectrics. Int. J. Fract. 165, $163-173$.

Xu, B.X., Schrade, D., Mueller, R., Gross, D., 2009. Micromechanical analysis of ferroelectric structures by a phase field method. Comput. Mater. Sci. 45, 832-836.

Xu, X.P., Needleman, A., 1994. Numerical simulations of fast crack-growth in brittle solids. J. Mech. Phys. Solids 42, $1397-1434$.

Yang, L., Dayal, K., 2011. Effect of lattice orientation, surface modulation, and applied fields on free-surface domain structure in ferroelectrics. Acta Mater. 59, 6594-6603.

Yang, L., Dayal, K., 2012. Microstructure and stray electric fields at surface cracks in ferroelectrics. Int. J. Fract. 174, $17-27$.

Yang, W., Zhu, T., 1998. Switch-toughening of ferroelectrics subjected to electric fields. J. Mech. Phys. Solids 46, 291-311.

Zhang, T.Y., Gao, C.F., 2004. Fracture behaviors of piezoelectric materials. Theor. Appl. Fract. Mech. 41, 339-379.

Zhang, W., Bhattacharya, K., 2005. A computational model of ferroelectric domains. Part i: model formulation and domain switching. Acta Mater. 53, 185-198.

Zhu, T., Yang, W., 1997. Toughness variation of ferroelectrics by polarization switch under non-uniform electric field. Acta Mater. 45, 4695-4702.

Zhu, T., Yang, W., 1999. Fatigue crack growth in ferroelectrics driven by cyclic electric loading. J. Mech. Phys. Solids 47, 81-97. 